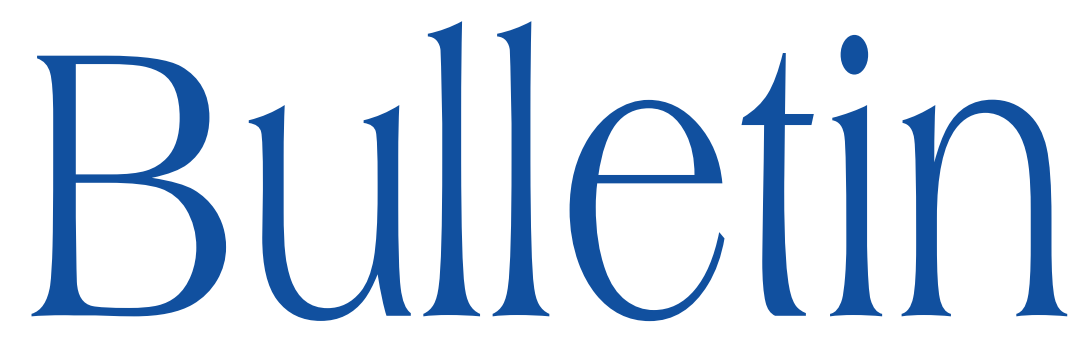

de la SOCIÉTÉ MATHÉMATIQUE DE FRANCE

\title{
GLOBAL EXISTENCE OF SOLUTIONS TO SCHRÖDINGER EQUATIONS ON COMPACT RIEMANNIAN MANIFOLDS BELOW $H^{1}$
}

Sijia Zhong

Tome 138

Fascicule 4

2010 


\title{
GLOBAL EXISTENCE OF SOLUTIONS TO SCHRÖDINGER EQUATIONS ON COMPACT RIEMANNIAN MANIFOLDS BELOW $H^{1}$
}

\author{
BY SiJiA ZHONG
}

\begin{abstract}
In this paper, we will study global well-posedness for the cubic defocusing nonlinear Schrödinger equations on the compact Riemannian manifold without boundary, below the energy space, i.e. $s<1$, under some bilinear Strichartz assumption. We will find some $\tilde{s}<1$, such that the solution is global for $s>\tilde{s}$.
\end{abstract}

RÉSumÉ (Existence globale de solutions des équations de Schrödinger sur les variétés riemanniennes compactes en régularité plus faible que $H^{1}$ )

Nous nous intéressons dans cet article au caractère bien posé des équations de Schrödinger non-linéaires cubiques défocalisantes sur les variétés riemanniennes compactes sans bord, en régularité $H^{s}, s<1$, sous certaines conditions bilinéaires de Strichartz. Nous trouvons un $\tilde{s}<1$ tel que la solution est globale pour $s>\tilde{s}$.

Texte reçu le 7 octobre 2008, révisé le 14 juillet 2009, accepté le 23 juillet 2009

Sijia Zhong, Department of Mathematics, Southeast University, Nanjing 210096, China. Laboratoire de Mathématique, Université Paris-sud 11, Bât 425, 91405 Orsay Cedex, France 2000 Mathematics Subject Classification. - 35Q55, 37K05, 37L50, 81Q20.

Key words and phrases. - Schrödinger equation, compact Riemannian manifold, global, I-method.

This work is supported by NSFC 10871175, NSFC 10926146, and NSFC 11001049. 


\section{Introduction}

Suppose $(M, g)$ is any compact Riemannian manifold of dimension 2, without boundary. In this paper, we will study the Cauchy problem for the cubic defocusing nonlinear Schrödinger equations posed on $M$,

$$
\left\{\begin{array}{l}
i u_{t}+\triangle u=|u|^{2} u \\
u(0, x)=u_{0}(x) \in H^{s}(M),
\end{array}\right.
$$

where the solution $u$ is a complex valued function on $\mathbb{R} \times M$, and $\triangle$ denotes the laplace operator associated to the metric $g$ on $M$.

There are two conservation laws:

$$
L^{2} \text {-mass } \int_{M}|u(t, x)|^{2} d x=\int_{M}\left|u_{0}(x)\right|^{2} d x,
$$

and

$$
\text { energy } E(u(t))=\frac{1}{2} \int_{M}|\nabla u(t)|_{g}^{2} d x+\frac{1}{4} \int_{M}|u(t, x)|^{4} d x=E_{0} .
$$

From [10], N. Burq, P. Gérard, and N. Tzvetkov proved that, if bilinear Strichartz estimate $\left(\mathscr{P}_{s_{0}}\right)$ (see Definition 1.1) is satisfied for some $0<s_{0}<1$, then the Cauchy problem (1.1) is locally well-posed on $H^{s}(M), s>s_{0}$. Thus, as a corollary, if $s \geqslant 1$, combining with the conservation of energy and $L^{2}$-mass, the solution is global. The question we are interested in is whether they are global for $s_{0}<s<1$.

First of all, let us see the situation on the whole space $\mathbb{R}^{2}$. In this case, equation (1.1) is $L^{2}$-critical. From [12], the solution is locally well-posed on $H^{s}\left(\mathbb{R}^{2}\right), s \geqslant 0$, and also by the conservation laws above, it is easy to get the global well-posedness for $s \geqslant 1$. Then, what about $s<1$ ?

In 1998, J. Bourgain, by decomposing the initial data into high frequence part and low frequence part, proved that for $\frac{2}{3}<s<1$, the solution is global. Then in 2002, the I-team (J. Colliander, M. Keel, G. Staffilani, H. Takaoka, and T. Tao), in [14] introduced the I-method, and improved it to be $\frac{4}{7}<s<1$. After that, by combining with the refined Morawetz estimate, the result has been improved little by little, and the best result known is $\frac{2}{5}<s<1$, (see [17], [13], and [15]). Meanwhile, in [15], the authors claimed that by the method available, $\frac{4}{13}$ could be achieved. Recently, in [19], R. Killip, T. Tao and M. Visan proved that, when the initial data is radial, the solution is global for $s \geqslant 0$, which is exactly the optimal one.

Now, let us see what happens on the compact Riemannian manifold without boundary. The first breakthrough was made by J. Bourgain in [5]. He proved that for $\mathbb{T}^{2}$, it is locally well posed for $s>0$. Then in [7], he claimed that for $s>\frac{2}{3}$, the solution would be global and this result was proved by D. De Silva, N. Pavlović, G. Staffilani, and N. Tzirakis in [16]. Recently, Akahori in 
[1] proved that for the compact manifold without boundary of dimension 2 , if $s>\frac{11-7 \alpha_{1}+4 \alpha_{2}}{\left(1-\alpha_{1}\right)+\left(11-7 \alpha_{1}+4 \alpha_{2}\right)}$, the solution of (1.1) would be global exists in $H^{s}(M)$, here $\left(\alpha_{1}, \alpha_{2}\right)$ is the pair of positive numbers satisfying

$$
\sharp\left\{k \in \mathbb{N}:\left|\sqrt{\lambda_{k}}-\mu\right| \leq A\right\} \leq C \mu^{\alpha_{1}} A^{\alpha_{2}} .
$$

For example for all the compact manifold without boundary, the above estimate holds at least with $\left(\alpha_{1}, \alpha_{2}\right)=(1,1)$. Particularly, for $\mathbb{T}^{2}$ and $\mathbb{S}^{2},\left(\alpha_{1}, \alpha_{2}\right)=$ $(0,1)$, and the $s$ corresponding to them is $s>\frac{15}{16}$.

Here, we are also interested in obtaining an abstract result.

Let us give the main condition of this paper, which is the bilinear Strichartz estimates.

DeFINITION 1.1. - Let $0 \leqslant s_{0}<1$. We say that $S(t)=e^{i t \Delta}$, the flow of the linear Schrödinger equation on $M$ stated above, satisfies property $\left(\mathscr{P}_{s_{0}}\right)$ if for all dyadic numbers $N, L$, and $u_{0}, v_{0} \in L^{2}(M)$ localized on dyadic intervals of order $N$, L respectively, i.e.

$$
\mathbf{1}_{N \leqslant \sqrt{-\triangle}<2 N}\left(u_{0}\right)=u_{0}, \text { and } \mathbf{1}_{L \leqslant \sqrt{-\triangle}<2 L}\left(v_{0}\right)=v_{0}
$$

the following estimate holds:

$$
\left\|S(t) u_{0} S(t) v_{0}\right\|_{L^{2}\left((0,1)_{t} \times M\right)} \leqslant C(\min (N, L))^{s_{0}}\left\|u_{0}\right\|_{L^{2}(M)}\left\|v_{0}\right\|_{L^{2}(M)} .
$$

In fact, such kind of bilinear Strichartz estimates were established and used by several authors in the context of the wave equations and of the Schrödinger equations. For example, in [10], N. Burq, P. Gérard, N. Tzvetkov showed that for Zoll surface of dimension 2, especially for $\mathbb{S}^{2}, s_{0}=\frac{1}{4}+$. Then in [11], they proved that $\left(\mathscr{P}_{s_{0}}\right)$ holds for $s_{0}=\frac{1}{2}+$ for $\mathbb{S}^{3}$ and $s_{0}=\frac{3}{4}+$ for $\mathbb{S}^{2} \times \mathbb{S}^{1}$. Also the results from J. Bourgain [5], [4], and [8] proved that $s_{0}=0+$ for $\mathbb{T}^{2}, s_{0}=\frac{1}{2}+$ for $\mathbb{T}^{3}$, and $s_{0}=\frac{2}{3}+$ for $\tilde{T}^{3}=\mathbb{R}^{3} / \prod_{j=1}^{3}\left(a_{j} \mathbb{Z}\right)$, where $a_{j}$ are pairwise irrational numbers. And R. Anton in [2] proved that $s_{0}=\frac{3}{4}+$ for general manifolds with boundary and manifolds without boundary equipped with a Lipschitz metric $g$ for dimension $d=2$, and for dimension 3 for the nontrapping case. Recently, this result is improved by M. D. Blair, H. F. Smith and C. D. Sogge in [3] to be $\frac{2}{3}+$.

Then, the main result of the paper is,

THEOREM 1.2. - Assume that there exists some $s_{0}<s<1$, such that condition $\left(\mathscr{P}_{s_{0}}\right)$ holds, then there must be some $s_{0}<\tilde{s}<1$, so that for $s>\tilde{s}$, the solution to the Cauchy problem (1.1) is global, here

$$
\tilde{s}=\frac{\sqrt{2}}{2}+\left(1-\frac{\sqrt{2}}{2}\right) s_{0} .
$$


Moreover,

$$
\|u(T)\|_{H^{s}} \lesssim T^{\frac{\left(s-s_{0}\right)(1-s)}{2 s^{2}-4 s_{0} s+s_{0}^{2}+2 s_{0}-1}}+\text { for } T \gg 1
$$

REMARK 1.3. - 1. $\tilde{s}$ is monotone increasing with respect to $s_{0}$, and for $s_{0} \rightarrow$ $1, \tilde{s} \rightarrow 1$.

2. For the special case $s_{0}=0+\left(\mathbb{T}^{2}\right), \tilde{s}=\frac{\sqrt{2}}{2} \sim 0.707$. And for $s_{0}=\frac{1}{4}+($ Zoll $)$, $\tilde{s}=\frac{2+3 \sqrt{2}}{8} \sim 0.78$.

3. When $s \rightarrow 1,\|u(T)\|_{H^{s}}$ is controlled by some constant.

Now, we will state the main idea for the proof briefly.

The aim is to imitate the $H^{1}$ argument with the energy. Hence we apply some smoothing operator to improve the regularity of the solution $u$, so that it makes sense for energy. However, the modified energy isn't conserved any more, so the crucial point is to estimate the growth of the energy. But, contrarily from the $\mathbb{R}^{2}$ case, the Fourier transformation couldn't be extended trivially. Although, we use eigenfunction expansion for $M$, there are still some obstacles, especially for the case high-high-low-low eigenvalues. Hence, we need to localized the function to some coordinate patch, and use some semiclassical analysis tools to deal with it.

The paper is organized as follows: in Section 2, we will give some notations and lemmas that will be used later, and in Section 3, we will prove the local well posedness for the modified equation. Then in Section 4, 5 and 6, the change of energy would be estimated, the results of which, will help to prove Theorem 1.2 in Section 7. Finally, in Section 8, which is also the appendix, we will prove two important lemmas that appear in the paper.

\section{Notations}

In this paper, we denote $s+$ for $s+\epsilon$, and $s-$ for $s-\epsilon$, with some constant $\epsilon>0$ small enough, and by $\left\langle\xi>,\left(1+|\xi|^{2}\right)^{\frac{1}{2}}\right.$.

$A \lesssim B$ means there is some constant $C$, such that $A \leqslant C B$, and $A \sim B$ means both $A \lesssim B$ and $B \lesssim A$.

As the spectrum of $\triangle$ is discrete, let $e_{k} \in L^{2}(M), k \in \mathbb{N}$, be an orthonormal basis of eigenfunctions of $-\triangle$ associated to eigenvalues $\mu_{k}$. Denote by $P_{k}$ the orthogonal projector on $e_{k}$. The following space is called Bourgain spaces:

TOME $138-2010-\mathrm{N}^{\mathrm{O}} 4$ 
Definition 2.1. - The space $X^{s, b}(\mathbb{R} \times M)$ is the completion of $C_{0}^{\infty}\left(\mathbb{R}_{t} ; H^{s}(M)\right)$ for the norm

$$
\begin{aligned}
\|u\|_{X^{s, b}(\mathbb{R} \times M)}^{2} & =\sum_{k}\left\|<\tau+\mu_{k}>^{b}<\mu_{k}>^{\frac{s}{2}} \widehat{P_{k} u}(\tau)\right\|_{L^{2}\left(\mathbb{R}_{t} ; L^{2}(M)\right)}^{2} \\
& =\left\|e^{-i t \triangle} u(t, \cdot)\right\|_{H^{b}\left(\mathbb{R}_{t} ; H^{s}\right)}^{2},
\end{aligned}
$$

where $\widehat{P_{k} u}(\tau)$ denotes the Fourier transform of $P_{k} u$ with respect to the time variable. And we denote it as $X^{s, b}$ when there is no confusion.

Then, for $1 \geqslant T>0$, we denote by $X_{T}^{s, b}(M)$ the space of restrictions of elements of $X^{s, b}(\mathbb{R} \times M)$ endowed with the norm

$$
\|u\|_{X_{T}^{s, b}}=\inf \left\{\|\tilde{u}\|_{X^{s, b}(\mathbb{R} \times M)},\left.\tilde{u}\right|_{(-T, T) \times M}=u\right\} .
$$

The following proposition, (see J. Ginibre [18], and N. Burq, P. Gérard, and N. Tzvetkov [10]), gathers basic properties of this space.

Proposition 2.2. - 1. $u \in X^{s, b}(\mathbb{R} \times M) \Longleftrightarrow e^{-i t \triangle} u(t, \cdot) \in H^{b}\left(\mathbb{R}, H^{s}(M)\right)$.

2. For $b>\frac{1}{2}, X^{s, b}(\mathbb{R} \times M) \hookrightarrow C\left(\mathbb{R}, H^{s}(M)\right)$, and $X_{T}^{s, b}(M) \hookrightarrow$ $C\left((-T, T), H^{s}(M)\right)$.

3. $X^{0, \frac{1}{4}}(\mathbb{R} \times M) \hookrightarrow L^{4}\left(\mathbb{R}, L^{2}(M)\right)$.

4. For $s_{1} \leqslant s_{2}$, and $b_{1} \leqslant b_{2}, X^{s_{2}, b_{2}}(\mathbb{R} \times M) \hookrightarrow X^{s_{1}, b_{1}}(\mathbb{R} \times M)$.

5. For $0 \leqslant b^{\prime}<b<\frac{1}{2},\|u\|_{X_{T}^{s, b^{\prime}}} \lesssim T^{b-b^{\prime}}\|u\|_{X_{T}^{s, b}}$.

Then, from Lemma 2.3 of [10], the condition $\left(\mathscr{P}_{s_{0}}\right)$ is equivalent to the following statement:

For any $b>\frac{1}{2}$, and any $f, g \in X^{0, b}(\mathbb{R} \times M)$ satisfying

$$
\mathbf{1}_{N \leqslant \sqrt{-\triangle}<2 N}(f)=f, \mathbf{1}_{L \leqslant \sqrt{-\triangle}<2 L}(g)=g,
$$

one has

$$
\|f g\|_{L^{2}(\mathbb{R} \times M)} \leqslant C(\min (N, L))^{s_{0}}\|f\|_{X^{0, b}(\mathbb{R} \times M)}\|g\|_{X^{0, b}(\mathbb{R} \times M)} .
$$

By some interpolation, there would be

LEMma 2.3. - If condition $\left(\mathscr{P}_{s_{0}}\right)$ holds, then for any $1 \geqslant s>s_{0}$, b, which satisfies $\frac{1}{4}<b^{\prime}(s)=\frac{1}{4}\left(1+\frac{1-s}{1-s_{0}}\right)+\leqslant b<\frac{1}{2}$, such that for any $f, g \in X^{0, b}(\mathbb{R} \times$ $M)$, satisfying

$$
\mathbf{1}_{N \leqslant \sqrt{-\triangle}<2 N}(f)=f, \text { and } \mathbf{1}_{L \leqslant \sqrt{-\triangle}<2 L}(g)=g,
$$

one has

$$
\|f g\|_{L^{2}(\mathbb{R} \times M)} \leqslant C(\min (N, L))^{s}\|f\|_{X^{0, b}(\mathbb{R} \times M)}\|g\|_{X^{0, b}(\mathbb{R} \times M)} .
$$


The proof of this lemma could be referred to [10] and [21].

Now let us give the definition of I operator.

Definition 2.4. - For $N \gg 1$, define some smooth operator $I_{N}$, such that

$$
I_{N} u=m_{N}(\triangle) u=m\left(N^{-2} \triangle\right) u,
$$

where

$$
m(\xi)= \begin{cases}1 & |\xi| \leqslant 1 \\ \left(\frac{1}{|\xi|}\right)^{\frac{1-s}{2}} & |\xi| \geqslant 2,\end{cases}
$$

is a smooth function. We denote $I$ for $I_{N}$ for simplicity when there is no confusion.

It is easy to check that

$$
\|u\|_{X^{s, \frac{1}{2}+}} \lesssim\left\|I_{N} u\right\|_{X^{1, \frac{1}{2}+}} \lesssim N^{1-s}\|u\|_{X^{s, \frac{1}{2}+}}
$$

and

$$
\|u\|_{H^{s}} \lesssim\left\|I_{N} u\right\|_{H^{1}} \lesssim N^{1-s}\|u\|_{H^{s}}
$$

\section{Local well posedness}

We impose the $I_{N}$ operator to both sides of the equation (1.1), and denote $v$ as $I_{N} u$, which satisfies the equation

$$
\left\{\begin{array}{l}
i \partial_{t} v+\triangle v=I_{N}\left(\left|I_{N}^{-1} v\right|^{2} I_{N}^{-1} v\right) \\
v(0)=v_{0} .
\end{array}\right.
$$

So $v_{0} \in H^{1}(M)\left(\left\|v_{0}\right\|_{H^{1}} \lesssim N^{1-s}\right.$ by (2.7)), and the following proposition ensures the local well posedness of (3.1).

Proposition 3.1. - Suppose $0 \leqslant s_{0}<1$, such that $\left(\mathscr{P}_{s_{0}}\right)$ holds. For $1>s>s_{0}$, and $v_{0} \in H^{1}(M)$, there is some $\delta=C\left\|v_{0}\right\|_{H^{1}}^{-\frac{2\left(1-s_{0}\right)}{s-s_{0}}}$ 之 $N^{-\frac{2\left(1-s_{0}\right)(1-s)}{s-s_{0}}-}$, such that (3.1) is locally well posed on $[0, \delta]$, and the solution $v \in C\left([0, \delta], H^{1}(M)\right)$, satisfies

$$
\left\|I_{N} u\right\|_{X_{\delta}^{1, \frac{1}{2}+}}=\|v\|_{X_{\delta}^{1, \frac{1}{2}+}} \lesssim\left\|v_{0}\right\|_{H^{1}}
$$

where $C$ is some constant that is independent on the time and initial data.

Proof. - To prove this proposition, we need the following lemma, the proof of which is in the appendix. 
LEMMA 3.2. - For $s<1, b^{\prime}(s-)=\frac{1}{4}\left(1+\frac{1-s}{1-s_{0}}\right)+$, and $u \in X_{\delta}^{s, b^{\prime}(s-)}$, there exists

$$
\left\|I_{N}\left(|u|^{2} u\right)\right\|_{X_{\delta}^{1,-b^{\prime}(s-)}} \lesssim\left\|I_{N} u\right\|_{X_{\delta}^{1, b^{\prime}(s-)}}^{3}, s_{0}<s<1 .
$$

From this lemma, we can see that $\left\|I_{N}\left(\left|I_{N}^{-1} v\right|^{2} I_{N}^{-1} v\right)\right\|_{X_{\delta}^{1,-b^{\prime}(s-)}} \lesssim\|v\|_{X_{\delta}^{1, b^{\prime}(s-)}}^{3}$. Then by Duhamel's formula, Proposition 2.11 in [10], and Proposition 2.2, we have

$$
\begin{aligned}
\|v\|_{X_{\delta}^{1, \frac{1}{2}+}} & \lesssim\left\|v_{0}\right\|_{H^{1}}+\delta^{1-\frac{1}{2}-b^{\prime}(s-)-}\left\|I_{N}\left(\left|I_{N}^{-1} v\right|^{2} I_{N}^{-1} v\right)\right\|_{X_{\delta}^{1,-b^{\prime}(s-)}} \\
& \lesssim\left\|v_{0}\right\|_{H^{1}}+\delta^{\frac{1}{2}-b^{\prime}(s-)-}\|v\|_{X_{\delta}^{1, b^{\prime}(s-)}}^{3} \\
& \lesssim\left\|v_{0}\right\|_{H^{1}}+\delta^{\frac{1}{2}-b^{\prime}(s-)-} \delta^{3 \times\left(\frac{1}{2}-b^{\prime}(s-)\right)-}\|v\|_{X_{\delta}^{1, \frac{1}{2}+}}^{3} \\
& \lesssim\left\|v_{0}\right\|_{H^{1}}+\delta^{\frac{s-s_{0}}{1-s_{0}}-}\|v\|_{X_{\delta}^{1, \frac{1}{2}+}}^{3} .
\end{aligned}
$$

Hence, by choosing $\delta \sim\left\|v_{0}\right\|_{H^{1}}^{-\frac{2\left(1-s_{0}\right)}{s-s_{0}}}-\gtrsim N^{-\frac{2\left(1-s_{0}\right)(1-s)}{s-s_{0}}}-$, and the standard contraction argument, we get the result of the proposition.

\section{The change of energy}

As $E(v(t))$ is not conserved any more, we have to calculate its variation on the time interval $[0, \delta]$.

Proposition 4.1. - For the solution $v$ to the Cauchy problem (3.1), its variation of energy on the time interval $[0, \delta]$ is

$E(v(\delta)) \leqslant E\left(v_{0}\right)+C\left(\left(N^{-\left(1-s_{0}\right)+} \delta^{\frac{1}{2}-}+N^{-2\left(1-s_{0}\right)+}\right)\left\|v_{0}\right\|_{H^{1}}^{4}+N^{-2+s_{0}+} \delta^{\frac{1}{2}-}\left\|v_{0}\right\|_{H^{1}}^{6}\right)$.

Proof. - Since

$$
\begin{aligned}
\partial_{t} E(v(t)) & =\operatorname{Re} \int_{M} \overline{\nabla v} \nabla v_{t} d x+\operatorname{Re} \int_{M}|v|^{2} \bar{v} v_{t} d x \\
& =-\operatorname{Re} \int_{M} \triangle \bar{v} v_{t} d x+\operatorname{Re} \int_{M}|v|^{2} \bar{v} v_{t} d x \\
& =\operatorname{Re} \int v_{t}\left(|v|^{2} \bar{v}-\triangle \bar{v}\right) d x
\end{aligned}
$$

BULLETIN DE LA SOCIÉtÉ MATHÉMATIQUE DE FRANCE 


$$
\begin{aligned}
= & \operatorname{Re} \int_{M} v_{t}\left(|v|^{2} \bar{v}-I_{N}\left(\left|I_{N}^{-1} v\right|^{2} \overline{I_{N}^{-1} v}\right)\right) d x \\
= & -\operatorname{Im} \int_{M}\left(\triangle v-I_{N}\left(\left|I_{N}^{-1} v\right|^{2} I_{N}^{-1} v\right)\right)\left(|v|^{2} \bar{v}-I_{N}\left(\left|I_{N}^{-1} v\right|^{2} \overline{I_{N}^{-1} v}\right)\right) d x \\
= & -\operatorname{Im} \int_{M} \triangle I_{N} u\left(\left|I_{N} u\right|^{2} \overline{I_{N} u}-I_{N}\left(|u|^{2} \bar{u}\right)\right) d x \\
& +\operatorname{Im} \int_{M} I_{N}\left(|u|^{2} u\right)\left(\left|I_{N} u\right|^{2} \overline{I_{N} u}-I_{N}\left(|u|^{2} \bar{u}\right)\right) d x,
\end{aligned}
$$

Integral on $[0, \delta]$, then

$$
\begin{aligned}
E(v(\delta))-E\left(v_{0}\right)= & -\operatorname{Im} \int_{0}^{\delta} \int_{M} \triangle I_{N} u\left(\left|I_{N} u\right|^{2} \overline{I_{N} u}-I_{N}\left(|u|^{2} \bar{u}\right)\right) d x d t \\
& +\operatorname{Im} \int_{0}^{\delta} \int_{M} I_{N}\left(|u|^{2} u\right)\left(\left|I_{N} u\right|^{2} \overline{I_{N} u}-I_{N}\left(|u|^{2} \bar{u}\right)\right) d x d t
\end{aligned}
$$

From now on, we will use the notation $I$ instead of $I_{N}$.

The goal for Proposition 4.1 would be achieved if we have the following two estimates, which will be proved in Section 5 and 6 separately.

$(4.2)=-\operatorname{Im} \int_{0}^{\delta} \int_{M} \triangle I_{N} u\left(\left|I_{N} u\right|^{2} \overline{I_{N} u}-I_{N}\left(|u|^{2} \bar{u}\right)\right) d x d t \lesssim\left(N^{-\left(1-s_{0}\right)+} \delta^{\frac{1}{2}-}+N^{-2\left(1-s_{0}\right)+}\right)\left\|v_{0}\right\|_{H^{1}}^{4}$,

and

$(4.3)=\operatorname{Im} \int_{0}^{\delta} \int_{M} I_{N}\left(|u|^{2} u\right)\left(\left|I_{N} u\right|^{2} \overline{I_{N} u}-I_{N}\left(|u|^{2} \bar{u}\right)\right) d x d t \lesssim N^{-2+s_{0}+} \delta^{\frac{1}{2}-}\left\|v_{0}\right\|_{H^{1}}^{6}$, where $s>\max \left\{\frac{2}{3}, \frac{1+s_{0}}{2}\right\}$.

\section{Proof of estimate 4.4}

Proof. - We have

$$
\begin{aligned}
(4.2)= & -\operatorname{Im} \int_{0}^{\delta} \int_{M} \nabla \overline{I u}\left(\nabla\left(|I u|^{2} I u\right)-I\left(\nabla\left(|u|^{2} u\right)\right)\right) \\
= & -2 \operatorname{Im} \int_{0}^{\delta} \int_{M} \nabla \overline{I u}\left(|I u|^{2} \nabla I u-I\left(|u|^{2} \nabla u\right)\right) \\
& -\operatorname{Im} \int_{0}^{\delta} \int_{M} \nabla \overline{I u}\left((I u)^{2} \nabla \overline{I u}-I\left(u^{2} \nabla \bar{u}\right)\right) \\
= & I+I I .
\end{aligned}
$$


5.1. Estimates for I. - The purpose of this subsection is to prove that

$$
\begin{aligned}
I \lesssim & \left(N^{-\left(1-s_{0}\right)+} \delta^{\frac{1}{2}-}+N^{-2\left(1-s_{0}\right)+}\right)\left\|v_{0}\right\|_{H^{1}}^{4} . \\
I= & -2 \operatorname{Im} \int_{0}^{\delta} \int_{M} \nabla \overline{I u}\left(|I u|^{2} \nabla I u-I\left(|u|^{2} \nabla u\right)\right) \\
= & 2 \operatorname{Im} \int_{0}^{\delta} \int_{M} \nabla \overline{I u} I\left(|u|^{2} \nabla u\right) \\
= & 2 \operatorname{Im} \int_{0}^{\delta} \int_{M} \nabla \overline{I u} I\left(\left|\chi_{1}\left(N^{-2+} \triangle\right) u\right|^{2} \nabla u\right) \\
& +\mathrm{III}
\end{aligned}
$$

with some smooth cut off function $\chi_{1}$, such that

$$
\chi_{1}(\xi)=\left\{\begin{array}{l}
1|\xi| \leqslant 1 \\
0|\xi| \geqslant 2
\end{array}\right.
$$

And III is the sum of the terms:

$$
\begin{aligned}
& 2 \operatorname{Im} \int_{0}^{\delta} \int \nabla \overline{I u} I\left(\chi_{1}\left(N^{-2+} \triangle\right) u\left(1-\chi_{1}\left(N^{-2+} \triangle\right)\right) \bar{u} \nabla u\right), \\
& 2 \operatorname{Im} \int_{0}^{\delta} \int \nabla \overline{I u} I\left(\chi_{1}\left(N^{-2+} \triangle\right) \bar{u}\left(1-\chi_{1}\left(N^{-2+} \triangle\right)\right) u \nabla u\right),
\end{aligned}
$$

and

$$
2 \operatorname{Im} \int_{0}^{\delta} \int_{M} \nabla \overline{I u} I\left(\left|\left(1-\chi_{1}\left(N^{-2+} \triangle\right)\right) u\right|^{2} \nabla u\right) .
$$

5.1.1. Study of (5.3). - We are going to show that

$$
(5.3)=2 \operatorname{Im} \int_{0}^{\delta} \int \nabla \overline{I u} I\left(\left|\chi_{1}\left(N^{-2+} \triangle\right) u\right|^{2} \nabla u\right) \lesssim N^{-1+s_{0}+} \delta^{\frac{1}{2}-}\left\|v_{0}\right\|_{H^{1}}^{4}
$$

Proof. - We have

$$
\begin{aligned}
(5.3)= & 2 \operatorname{Im} \int_{0}^{\delta} \int \nabla \overline{I u} I\left(\left|\chi_{1}\left(N^{-2+} \triangle\right) u\right|^{2} \nabla u\right) \\
= & 2 \operatorname{Im} \int_{0}^{\delta} \int \nabla \overline{I u}\left|\chi_{1}\left(N^{-2+} \triangle\right) u\right|^{2} \nabla I u \\
& +2 \operatorname{Im} \int_{0}^{\delta} \int \nabla \overline{I u}\left[I,\left|\chi_{1}\left(N^{-2+} \triangle\right) u\right|^{2}\right] \nabla u
\end{aligned}
$$

BULLETIN DE LA SOCIÉTÉ MATHÉMATIQUE DE FRANCE 


$$
\begin{aligned}
& =2 \operatorname{Im} \int_{0}^{\delta} \int \nabla \overline{I u}\left[I,\left|\chi_{1}\left(N^{-2+} \triangle\right) u\right|^{2}\right] I^{-1} \nabla I u \\
& =2 \operatorname{Im} \int_{0}^{\delta} \int \nabla \overline{I u}[I, g] I^{-1} \nabla I u,
\end{aligned}
$$

with $g(x)=\left|\chi_{1}\left(N^{-2+} \triangle\right) u\right|^{2}$.

By partition of unity, it is enough to estimate $2 \operatorname{Im} \int_{0}^{\delta} \int \nabla \overline{I u} \chi_{2}\left([I, g] I^{-1} \nabla I u\right)$, where $\chi_{2} \in C_{0}^{\infty}(M)$ is supported in a coordinate patch.

Then (5.5) follows from the following lemma,

Lemma 5.1. - For the $u, g$, and $\chi_{2}$ appearing above, we have,

$2 \operatorname{Im} \int_{0}^{\delta} \int \nabla \overline{I u} \chi_{2}\left([I, g] I^{-1} \nabla I u\right) \lesssim N^{-1+s_{0}+} \delta^{\frac{1}{2}-}\|I u\|_{X^{1, \frac{1}{2}+}}^{4} \lesssim N^{-1+s_{0}+} \delta^{\frac{1}{2}-}\left\|v_{0}\right\|_{H^{1}}^{4}$.

We will postpone the proof of this lemma in the appendix (see section 8.2).

5.1.2. Estimate of III. - For III, we are going to prove that

$$
\mathrm{III} \lesssim\left(N^{-\left(1-s_{0}\right)+} \delta^{\frac{1}{2}-}+N^{-2\left(1-s_{0}\right)+}\right)\left\|v_{0}\right\|_{H^{1}}^{4} .
$$

Proof. - Because $\left\{e_{k}\right\}$ is an orthonomal basis of eigenfunction of $-\triangle$,

$\mathrm{III} \sim 2 \operatorname{Im} \sum_{N_{0}, N_{1}, N_{2}, N_{3}} \int_{0}^{\delta} \int \nabla \overline{I u}^{N_{0}} \frac{m_{N}\left(N_{0}^{2}\right)}{m_{N}\left(N_{1}^{2}\right) m_{N}\left(N_{2}^{2}\right) m_{N}\left(N_{3}^{2}\right)} I u^{N_{1}} \overline{I u}^{N_{2}} \nabla I u^{N_{3}} d x d t$, with

$$
u^{N_{j}}=\sum_{N_{j} \leqslant<\mu_{k}>\frac{1}{2}<2 N_{j}} P_{k} u, j=0,1,2,3,
$$

and $N_{1}$ or $N_{2} \gtrsim N^{1-}$.

Let

$$
I(N)=\left|\int_{0}^{\delta} \int \frac{m_{N}\left(N_{0}^{2}\right)}{m_{N}\left(N_{1}^{2}\right) m_{N}\left(N_{2}^{2}\right) m_{N}\left(N_{3}^{2}\right)} \nabla \overline{I u}^{N_{0}} I u^{N_{1}} I \bar{u}^{N_{2}} \nabla I u^{N_{3}} d x d t\right| .
$$

We will prove that part of the gradient of $\nabla I u^{N_{3}}$ could be shared by $I u^{N_{1}}$ and $I u^{N_{2}}$.

By symmetry argument and because the presence of complex conjugates will play no role here, we can assume $N_{1} \geqslant N_{2}$. So $\max \left\{N_{0}, N_{1}, N_{2}, N_{3}\right\} \gtrsim N^{1-}$, 
and by Lemma 2.6 of [10], we can see that there exists $\tilde{C}>0$ such that if $N_{i} \geqslant \tilde{C} \sum_{j \neq i}\left(N_{j}\right)$, then for every $p>0$, there exists

$$
\begin{aligned}
I(N) & \lesssim N_{i}^{-p} \max \left\{1,\left(\frac{N_{i}}{N}\right)\right\}^{3(1-s)} \int_{0}^{\delta}\left\|\nabla I u^{N_{0}}\right\|_{L_{x}^{2}}\left\|I u^{N_{1}}\right\|_{L_{x}^{2}}\left\|I u^{N_{2}}\right\|_{L_{x}^{2}}\left\|\nabla I u^{N_{3}}\right\|_{L_{x}^{2}} d t \\
& \lesssim N_{i}^{-p+3(1-s)} \prod_{j=0}^{3}\left\|<\nabla>I u^{N_{j}}\right\|_{L_{t}^{4} L_{x}^{2}} \\
& \lesssim N_{i}^{-p+3(1-s)} \prod_{j=0}^{3}\left\|<\nabla>I u^{N_{j}}\right\|_{X_{\delta}^{0, \frac{1}{4}}}^{4} \\
& \lesssim N_{i}^{-p+3(1-s)} \delta^{1-}\|I u\|_{X_{\delta}^{1, \frac{1}{2}+}}^{4} \\
& \lesssim N_{i}^{0-} N^{-(p-3(1-s))+} \delta^{1-}\|I u\|_{X_{\delta}^{1, \frac{1}{2}+}}^{4} \\
(5.10) & \lesssim N_{i}^{0-} N^{-(p-3(1-s))+} \delta^{1-}\left\|v_{0}\right\|_{H^{1}}^{4} .
\end{aligned}
$$

Hence, divide (5.9) into two parts, $J_{1}$ and $J_{2}$, where the summation is restricted to $N_{i} \geqslant \tilde{C} \sum_{j \neq i} N_{j}$ in $J_{1}$ and other possibilities are in $J_{2}$. Thus for $J_{1}$, as $N_{j}(j \neq i)$ could be controlled by $N_{i}$, we can choose $p$ large enough such that $J_{1}$ could be controlled by (5.5), hence we just need to deal with $J_{2}$.

(a) $N_{0}, N_{1}, N_{2}, N_{3} \ll N$.

Hence $\frac{m_{N}\left(N_{0}^{2}\right)}{m_{N}\left(N_{1}^{2}\right) m_{N}\left(N_{2}^{2}\right) m_{N}\left(N_{3}^{2}\right)}=1$.

By Lemma 2.3 and Proposition 2.2,

$$
\begin{aligned}
I(N) & \lesssim\left\|\nabla I u^{N_{0}} I u^{N_{2}}\right\|_{L_{t, x}^{2}}\left\|I u^{N_{1}} \nabla I u^{N_{3}}\right\|_{L_{t, x}^{2}} \\
& \lesssim N_{1}^{s_{0}+} N_{2}^{1-}\left\|\nabla I u^{N_{0}}\right\|_{X_{\delta}^{0, \frac{1}{4}+}}\left\|I u^{N_{2}}\right\|_{X_{\delta}^{0, \frac{1}{4}+}}\left\|I u^{N_{1}}\right\|_{X_{\delta}^{0, \frac{1}{2}+}}\left\|\nabla I u^{N_{3}}\right\|_{X_{\delta}^{0, \frac{1}{2}+}} \\
& \lesssim N_{1}^{s_{0}-1+} N_{2}^{0-} \delta^{2 \times\left(\frac{1}{2}-\frac{1}{4}\right)-} \prod_{i=0}^{3}\left\|I u^{N_{i}}\right\|_{X_{\delta}^{1, \frac{1}{2}+}} \\
& \lesssim N_{1}^{-\left(1-s_{0}\right)+} N_{2}^{0-} \delta^{\frac{1}{2}-} \prod_{i=0}^{3}\left\|I u^{N_{i}}\right\|_{X_{\delta}^{1, \frac{1}{2}+} \cdot}
\end{aligned}
$$

If $N_{3} \lesssim N_{1}$, then $N_{1} \gtrsim N^{1-}, N_{0} \lesssim N_{1}$ and

$$
\begin{aligned}
\sum_{N_{0}, N_{1}, N_{2}, N_{3}} I(N) \lesssim \sum_{N_{0}, N_{1}, N_{2}, N_{3}} N_{1}^{-\left(1-s_{0}\right)+} N_{0}^{0-} & N_{1}^{0-} N_{2}^{0-} N_{3}^{0-} \delta^{\frac{1}{2}-} \prod_{i=0}^{3}\left\|I u^{N_{i}}\right\|_{X_{\delta}^{1, \frac{1}{2}+}} \\
& \lesssim N^{-\left(1-s_{0}\right)+} \delta^{\frac{1}{2}-}\|I u\|_{X_{\delta}^{1, \frac{1}{2}+}}^{4}
\end{aligned}
$$


If $N_{3} \gg N_{1} \geqslant N_{2}$, then $N_{1} \gtrsim N^{1-}, N_{0} \sim N_{3}$,

$$
\begin{aligned}
& \sum_{N_{0}, N_{1}, N_{2}, N_{3}} I(N) \lesssim N^{-\left(1-s_{0}\right)+} \delta^{\frac{1}{2}-}\|I u\|_{X_{\delta}^{1, \frac{1}{2}+}}^{2} \sum_{N_{0} \sim N_{3}}\left\|I u^{N_{0}}\right\|_{X_{\delta}^{1, \frac{1}{2}+}}\left\|I u^{N_{3}}\right\|_{X_{\delta}^{1, \frac{1}{2}+}}
\end{aligned}
$$

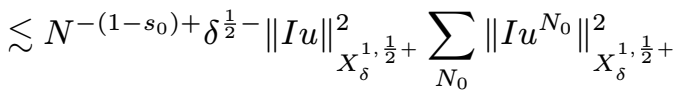

$$
\begin{aligned}
& \lesssim N^{-\left(1-s_{0}\right)+} \delta^{\frac{1}{2}-}\|I u\|_{X_{\delta}^{1, \frac{1}{2}+}}^{4} \cdot
\end{aligned}
$$

Combining (5.12) with (5.13), we have

$$
\sum_{N_{0}, N_{1}, N_{2}, N_{3}} I(N) \lesssim N^{-\left(1-s_{0}\right)+} \delta^{\frac{1}{2}-}\|I u\|_{X_{\delta}^{1, \frac{1}{2}+}}^{4} \lesssim N^{-\left(1-s_{0}\right)+} \delta^{\frac{1}{2}-}\left\|v_{0}\right\|_{H^{1}}^{4} .
$$

(b) At least one of $N_{i} \gtrsim N$.

(b1) $N_{1} \geqslant N_{3}$.

Hence, $N_{0} \lesssim N_{1}$, and $N_{1} \gtrsim N$.

(b11) $N_{1} \gtrsim N \gg N_{2}, N_{3}$.

In this case, $N_{1} \sim N_{0} \gtrsim N$, which gives $\frac{m_{N}\left(N_{0}^{2}\right)}{m_{N}\left(N_{1}^{2}\right) m_{N}\left(N_{2}^{2}\right) m_{N}\left(N_{3}^{2}\right)}=\frac{m_{N}\left(N_{0}^{2}\right)}{m_{N}\left(N_{1}^{2}\right)} \lesssim$ 1 , the left steps are the same as (a), and the result would even be better.

(b12) $N_{1} \geqslant N_{3} \gtrsim N \gg N_{2}$.

$$
\frac{m_{N}\left(N_{0}^{2}\right)}{m_{N}\left(N_{1}^{2}\right) m_{N}\left(N_{2}^{2}\right) m_{N}\left(N_{3}^{2}\right)} \lesssim N^{-2(1-s)}\left(N_{1} N_{3}\right)^{1-s} .
$$

By Lemma 2.3 and Proposition 2.2,

$$
\begin{aligned}
I(N) \lesssim & N^{-2(1-s)}\left(N_{1} N_{3}\right)^{1-s}\left\|\nabla I u^{N_{0}} I u^{N_{2}}\right\|_{L_{t, x}^{2}}\left\|I u^{N_{1}} \nabla I u^{N_{3}}\right\|_{L_{t, x}^{2}} \\
\lesssim & N^{-2(1-s)}\left(N_{1} N_{3}\right)^{1-s} N_{2}^{1-} N_{3}^{s_{0}+} \\
& \cdot\left\|\nabla I u^{N_{0}}\right\|_{X_{\delta}^{0, \frac{1}{4}+}}\left\|I u^{N_{2}}\right\|_{X_{\delta}^{0, \frac{1}{4}+}}\left\|I u^{N_{1}}\right\|_{X_{\delta}^{0, \frac{1}{2}+}}\left\|\nabla I u^{N_{3}}\right\|_{X_{\delta}^{0, \frac{1}{2}+}} \\
\lesssim & \delta^{\frac{1}{2}-} N^{-2(1-s)} N_{1}^{-s} N_{2}^{0-} N_{3}^{1-s+s_{0}+} \prod_{i=0}^{3}\left\|I u^{N_{i}}\right\|_{X_{\delta}^{1, \frac{1}{2}+}} \\
\lesssim & \delta^{\frac{1}{2}-} N^{-2(1-s)} N_{1}^{1-2 s+s_{0}+} N_{0}^{0-} N_{2}^{0-} N_{3}^{0-} \prod_{i=0}^{3}\left\|I u^{N_{i}}\right\|_{X_{\delta}^{1, \frac{1}{2}+}},
\end{aligned}
$$

then

$$
\sum I(N) \lesssim \delta^{\frac{1}{2}-} N^{-1+s_{0}+}\|I u\|_{X_{\delta}^{1, \frac{1}{2}+}}^{4} \lesssim \delta^{\frac{1}{2}-} N^{-1+s_{0}+}\left\|v_{0}\right\|_{H^{1}}^{4},
$$

with $s>\frac{1+s_{0}}{2}$. 
(b13) $N_{1} \geqslant N_{2} \gtrsim N \gg N_{3}$.

$$
\frac{m_{N}\left(N_{0}^{2}\right)}{m_{N}\left(N_{1}^{2}\right) m_{N}\left(N_{2}^{2}\right) m_{N}\left(N_{3}^{2}\right)} \lesssim N^{-2(1-s)}\left(N_{1} N_{2}\right)^{1-s} \text {. }
$$

Similarly,

$$
\begin{aligned}
I(N) \lesssim & N^{-2(1-s)}\left(N_{1} N_{2}\right)^{1-s}\left(N_{2} N_{3}\right)^{s_{0}+} \\
& \cdot\left\|\nabla I u^{N_{0}}\right\|_{X_{\delta}^{0, \frac{1}{2}+}}\left\|I u^{N_{1}}\right\|_{X_{\delta}^{0, \frac{1}{2}+}}\left\|I u^{N_{2}}\right\|_{X_{\delta}^{0, \frac{1}{2}+}}\left\|\nabla I u^{N_{3}}\right\|_{X_{\delta}^{0, \frac{1}{2}+}} \\
\lesssim & N^{-2(1-s)} N_{1}^{-s} N_{2}^{-s+s_{0}+} N_{3}^{s_{0}+} \prod_{i=0}^{3}\left\|I u^{N_{i}}\right\|_{X_{\delta}^{1, \frac{1}{2}+}} \\
\lesssim & N^{-2(1-s)} N_{1}^{-s+s_{0}+} N_{2}^{-s+s_{0}+} N_{0}^{0-} N_{3}^{0-} \prod_{i=0}^{3}\left\|I u^{N_{i}}\right\|_{X_{\delta}^{1, \frac{1}{2}+}}
\end{aligned}
$$

so

$$
\sum I(N) \lesssim N^{-2\left(1-s_{0}\right)+}\|I u\|_{X_{\delta}^{1, \frac{1}{2}+}}^{4} \lesssim N^{-2\left(1-s_{0}\right)+}\left\|v_{0}\right\|_{H^{1}}^{4}
$$

(b14) $N_{1} \geqslant N_{2}, N_{3} \gtrsim N$.

$$
\frac{m_{N}\left(N_{0}^{2}\right)}{m_{N}\left(N_{1}^{2}\right) m_{N}\left(N_{2}^{2}\right) m_{N}\left(N_{3}^{2}\right)} \lesssim N^{-3(1-s)}\left(N_{1} N_{2} N_{3}\right)^{1-s} \text {. }
$$

Also

$$
\begin{aligned}
I(N) \lesssim & N^{-3(1-s)}\left(N_{1} N_{2} N_{3}\right)^{1-s}\left(N_{2} N_{3}\right)^{s_{0}+} \\
& \cdot\left\|\nabla I u^{N_{0}}\right\|_{X_{\delta}^{0, \frac{1}{2}+}}\left\|I u^{N_{1}}\right\|_{X_{\delta}^{0, \frac{1}{2}+}}\left\|I u^{N_{2}}\right\|_{X_{\delta}^{0, \frac{1}{2}+}}\left\|\nabla I u^{N_{3}}\right\|_{X_{\delta}^{0, \frac{1}{2}+}} \\
\lesssim & N^{-3(1-s)} N_{1}^{-s} N_{2}^{-s+s_{0}+} N_{3}^{1-s+s_{0}+} \prod_{i=0}^{3}\left\|I u^{N_{i}}\right\|_{X_{\delta}^{1, \frac{1}{2}+}} \\
\lesssim & N^{-3(1-s)} N_{1}^{1-2 s+s_{0}+} N_{2}^{-s+s_{0}+} N_{0}^{0-} N_{3}^{0-} \prod_{i=0}^{3}\left\|I u^{N_{i}}\right\|_{X_{\delta}^{1, \frac{1}{2}+}}
\end{aligned}
$$

and

$$
\sum I(N) \lesssim N^{-2\left(1-s_{0}\right)+}\|I u\|_{X_{\delta}^{1, \frac{1}{2}+}}^{4} \lesssim N^{-2\left(1-s_{0}\right)+}\left\|v_{0}\right\|_{H^{1}}^{4}
$$

(b2) $N_{3} \geqslant N_{1} \geqslant N_{2}$.

In this case, $N_{3} \gtrsim N$, and $N_{0} \lesssim N_{3}$.

(b21) $N_{3} \gtrsim N \gg N_{1} \gtrsim N^{1-}$.

So $N_{0} \sim N_{3} \gtrsim N$, and

$$
\frac{m_{N}\left(N_{0}^{2}\right)}{m_{N}\left(N_{1}^{2}\right) m_{N}\left(N_{2}^{2}\right) m_{N}\left(N_{3}^{2}\right)} \sim 1,
$$

BULLETIN DE LA SOCIÉTÉ MATHÉMATIQUE DE FRANCE 
So this case can be dealt by the same way as case (a), and the result is the same.

(b22) $N_{3} \geqslant N_{1} \gtrsim N \gg N_{2}$.

(b221) $N_{3} \sim N_{1}$.

$$
\frac{m_{N}\left(N_{0}^{2}\right)}{m_{N}\left(N_{1}^{2}\right) m_{N}\left(N_{2}^{2}\right) m_{N}\left(N_{3}^{2}\right)} \lesssim N^{-2(1-s)}\left(N_{1} N_{3}\right)^{1-s},
$$

and

$$
\begin{aligned}
I(N) \lesssim & N^{-2(1-s)}\left(N_{1} N_{3}\right)^{1-s} N_{1}^{s_{0}+} N_{2}^{1-} \\
& \cdot\left\|\nabla I u^{N_{0}}\right\|_{X_{\delta}^{0, \frac{1}{2}+}}\left\|I u^{N_{1}}\right\|_{X_{\delta}^{0, \frac{1}{2}+}}\left\|I u^{N_{2}}\right\|_{X_{\delta}^{0, \frac{1}{4}+}}\left\|\nabla I u^{N_{3}}\right\|_{X_{\delta}^{0, \frac{1}{4}+}} \\
\lesssim & N^{-2(1-s)} N_{1}^{-s+s_{0}+} N_{2}^{0-} N_{3}^{1-s} \delta^{\frac{1}{2}-} \prod_{i=0}^{3}\left\|I u^{N_{i}}\right\|_{X_{\delta}^{1, \frac{1}{2}+}} \\
\sim & N^{-2(1-s)} N_{3}^{1-2 s+s_{0}+} N_{2}^{0-} \delta^{\frac{1}{2}-} \prod_{i=0}^{3}\left\|I u^{N_{i}}\right\|_{X_{\delta}^{1, \frac{1}{2}+}}
\end{aligned}
$$

Hence,

$$
\sum I(N) \lesssim N^{-1+s_{0}+} \delta^{\frac{1}{2}-}\|I u\|_{X_{\delta}^{1, \frac{1}{2}+}}^{4} \lesssim N^{-1+s_{0}+} \delta^{\frac{1}{2}-}\left\|v_{0}\right\|_{H^{1}}^{4} .
$$

(b222) $N_{1} \ll N_{3}$.

So $N_{0} \sim N_{3}$ and

$$
\frac{m_{N}\left(N_{0}^{2}\right)}{m_{N}\left(N_{1}^{2}\right) m_{N}\left(N_{2}^{2}\right) m_{N}\left(N_{3}^{2}\right)} \lesssim N^{-(1-s)} N_{1}^{1-s} .
$$

Then

$$
\begin{aligned}
I(N) \lesssim & N^{-(1-s)} N_{1}^{1-s} N_{1}^{s_{0}+} N_{2}^{1-} \\
& \cdot\left\|\nabla I u^{N_{0}}\right\|_{X_{\delta}^{0, \frac{1}{2}+}}\left\|I u^{N_{1}}\right\|_{X_{\delta}^{0, \frac{1}{2}+}}\left\|I u^{N_{2}}\right\|_{X_{\delta}^{0, \frac{1}{4}+}}\left\|\nabla I u^{N_{3}}\right\|_{X_{\delta}^{0, \frac{1}{4}+}} \\
\lesssim & N^{-(1-s)} N_{1}^{-s+s_{0}+} N_{2}^{0-} \delta^{\frac{1}{2}-} \prod_{i=0}^{3}\left\|I u^{N_{i}}\right\|_{X_{\delta}^{1, \frac{1}{2}+} \cdot}
\end{aligned}
$$

And

$$
\begin{aligned}
& \sum_{N_{0}, N_{1}, N_{2}, N_{3}} I(N) \lesssim N^{-1+s_{0}+} \delta^{\frac{1}{2}-}\|I u\|_{X_{\delta}^{1, \frac{1}{2}+}}^{2} \sum_{N_{0} \sim N_{3}}\left\|I u^{N_{0}}\right\|_{X_{\delta}^{1, \frac{1}{2}+}}\left\|I u^{N_{3}}\right\|_{X_{\delta}^{1, \frac{1}{2}+}} \\
& \lesssim N^{-1+s_{0}+} \delta^{\frac{1}{2}-}\|I u\|_{X_{\delta}^{1, \frac{1}{2}+}}^{2} \sum_{N_{0}}\left\|I u^{N_{0}}\right\|_{X_{\delta}^{1, \frac{1}{2}+}}^{2} \\
& \lesssim N^{-1+s_{0}+} \delta^{\frac{1}{2}-}\|I u\|_{X_{\delta}^{1, \frac{1}{2}+}}^{4} \\
& \lesssim N^{-1+s_{0}+} \delta^{\frac{1}{2}-}\left\|v_{0}\right\|_{H^{1}}^{4}
\end{aligned}
$$

TOME $138-2010-\mathrm{N}^{\circ} 4$ 
(b23) $N_{3} \geqslant N_{1} \geqslant N_{2} \gtrsim N$.

Also divide into two cases, $N_{1} \sim N_{3}$ and $N_{1} \ll N_{3}$.

First, if $N_{1} \sim N_{3}$,

$$
\frac{m_{N}\left(N_{0}^{2}\right)}{m_{N}\left(N_{1}^{2}\right) m_{N}\left(N_{2}^{2}\right) m_{N}\left(N_{3}^{2}\right)} \lesssim N^{-3(1-s)}\left(N_{1} N_{2} N_{3}\right)^{1-s},
$$

and

$$
\begin{aligned}
I(N) \lesssim & N^{-3(1-s)}\left(N_{1} N_{2} N_{3}\right)^{1-s}\left(N_{0} N_{2}\right)^{s_{0}+} \\
& \cdot\left\|\nabla I u^{N_{0}}\right\|_{X_{\delta}^{0, \frac{1}{2}+}}\left\|I u^{N_{1}}\right\|_{X_{\delta}^{0, \frac{1}{2}+}}\left\|I u^{N_{2}}\right\|_{X_{\delta}^{0, \frac{1}{2}+}}\left\|\nabla I u^{N_{3}}\right\|_{X_{\delta}^{0, \frac{1}{2}+}} \\
\lesssim & N^{-3(1-s)} N_{1}^{-s+s_{0}+} N_{2}^{-s+s_{0}+} N_{3}^{1-s} \prod_{i=0}^{3}\left\|I u^{N_{i}}\right\|_{X_{\delta}^{1, \frac{1}{2}+}} \\
\lesssim & N^{-3(1-s)} N_{3}^{1-2 s+s_{0}+} N_{2}^{-s+s_{0}+} \prod_{i=0}^{3}\left\|I u^{N_{i}}\right\|_{X_{\delta}^{1, \frac{1}{2}+}} \cdot
\end{aligned}
$$

So

$$
\sum I(N) \lesssim N^{-2+2 s_{0}+}\|I u\|_{X_{\delta}^{1, \frac{1}{2}+}}^{4} \lesssim N^{-2+2 s_{0}+}\left\|v_{0}\right\|_{H^{1}}^{4}
$$

For the second case, we have $N_{3} \sim N_{0} \gtrsim N$,

$$
\frac{m_{N}\left(N_{0}^{2}\right)}{m_{N}\left(N_{1}^{2}\right) m_{N}\left(N_{2}^{2}\right) m_{N}\left(N_{3}^{2}\right)} \lesssim N^{-2(1-s)}\left(N_{1} N_{2}\right)^{1-s},
$$

and

$$
\begin{aligned}
I(N) \lesssim & N^{-2(1-s)}\left(N_{1} N_{2}\right)^{1-s}\left(N_{1} N_{2}\right)^{s_{0}+} \\
& \cdot\left\|\nabla I u^{N_{0}}\right\|_{X_{\delta}^{0, \frac{1}{2}+}}\left\|I u^{N_{1}}\right\|_{X_{\delta}^{0, \frac{1}{2}+}}\left\|I u^{N_{2}}\right\|_{X_{\delta}^{0, \frac{1}{2}+}}\left\|\nabla I u^{N_{3}}\right\|_{X_{\delta}^{0, \frac{1}{2}+}} \\
\lesssim & N_{1}^{-s+s_{0}+} N_{2}^{-s+s_{0}+} N^{-2(1-s)} \prod_{i=0}^{3}\left\|I u^{N_{i}}\right\|_{X_{\delta}^{1, \frac{1}{2}+}},
\end{aligned}
$$

which gives

$$
\sum_{N_{0}, N_{1}, N_{2}, N_{3}} I(N) \lesssim N^{-2+2 s_{0}+}\|I u\|_{X_{\delta}^{1, \frac{1}{2}+}}^{4} \lesssim N^{-2+2 s_{0}+}\left\|v_{0}\right\|_{H^{1}}^{4},
$$

by the same arguments as (b222).

Conclusively,

$$
\text { III } \lesssim\left(N^{-\left(1-s_{0}\right)+} \delta^{\frac{1}{2}-}+N^{-2\left(1-s_{0}\right)+}\right)\left\|v_{0}\right\|_{H^{1}}^{4} .
$$

Combining with the result of section 5.1.1,

$$
I \lesssim\left(N^{-\left(1-s_{0}\right)+} \delta^{\frac{1}{2}-}+N^{-2\left(1-s_{0}\right)+}\right)\left\|v_{0}\right\|_{H^{1}}^{4} .
$$


5.2. Estimates for II. - Because

$$
\begin{aligned}
\mathrm{II} & =-\operatorname{Im} \int_{0}^{\delta} \int_{M} \nabla \overline{I u}\left((I u)^{2} \nabla \overline{I u}-I\left(u^{2} \nabla \bar{u}\right)\right) \\
& =-\operatorname{Im} \sum_{N_{0}, N_{1}, N_{2}, N_{3}} \int_{0}^{\delta} \int_{M} \nabla \overline{I u}^{N_{0}}\left(1-\frac{m_{N}\left(N_{0}^{2}\right)}{m_{N}\left(N_{1}^{2}\right) m_{N}\left(N_{2}^{2}\right) m_{N}\left(N_{3}^{2}\right)}\right) I u^{N_{1}} I u^{N_{2}} \nabla \overline{I u}^{N_{3}},
\end{aligned}
$$

and $\left|1-\frac{m_{N}\left(N_{0}^{2}\right)}{m_{N}\left(N_{1}^{2}\right) m_{N}\left(N_{2}^{2}\right) m_{N}\left(N_{3}^{2}\right)}\right| \lesssim \frac{m_{N}\left(N_{0}^{2}\right)}{m_{N}\left(N_{1}^{2}\right) m_{N}\left(N_{2}^{2}\right) m_{N}\left(N_{3}^{2}\right)}$, all the other cases could be estimated the same as I, except for

$$
-\operatorname{Im} \int_{0}^{\delta} \int_{M} \nabla \overline{I u}\left(\left(\chi_{1}\left(N^{-2+} \triangle\right) I u\right)^{2} \nabla \overline{I u}-I\left(\left(\chi_{1}\left(N^{-2+} \triangle\right) u\right)^{2} \nabla \bar{u}\right)\right) .
$$

As

$$
\begin{aligned}
(5.27) & =-\operatorname{Im} \int_{0}^{\delta} \int_{M} \nabla \overline{I u}\left(\left(\chi_{1}\left(N^{-2+} \triangle\right) I u\right)^{2} \nabla \overline{I u}-I\left(\left(\chi_{1}\left(N^{-2+} \triangle\right) u\right)^{2} \nabla \bar{u}\right)\right) \\
& =-\operatorname{Im} \int_{0}^{\delta} \int \nabla \overline{I u}\left(\left(\chi_{1}\left(N^{-2+} \triangle\right) u\right)^{2} \nabla \overline{I u}-\left(\chi_{1}\left(N^{-2+} \triangle\right) u\right)^{2} \nabla \overline{I u}-[I, g] I^{-1} \nabla \overline{I u}\right) \\
& =\operatorname{Im} \int_{0}^{\delta} \int \nabla \overline{I u}[I, g] I^{-1} \nabla \overline{I u}
\end{aligned}
$$

where $\underline{g}=\left(\chi_{1}\left(N^{-2+} \triangle\right) u\right)^{2}$.

From the proof of section 5.1.1 and Lemma 5.1, we can see that the presence of complex conjugates makes no difference there, hence we have

$$
(5.27) \lesssim N^{-1+s_{0}+} \delta^{\frac{1}{2}-}\|I u\|_{X_{\delta}^{1, \frac{1}{2}+}}^{4} \lesssim N^{-1+s_{0}+} \delta^{\frac{1}{2}-}\left\|v_{0}\right\|_{H^{1}}^{4} .
$$

So

$$
I I \lesssim\left(N^{-\left(1-s_{0}\right)+} \delta^{\frac{1}{2}-}+N^{-2\left(1-s_{0}\right)+}\right)\left\|v_{0}\right\|_{H^{1}}^{4}
$$

Taking section 5.1 into consideration, it has

$$
(4.2) \lesssim\left(N^{-\left(1-s_{0}\right)+} \delta^{\frac{1}{2}-}+N^{-2\left(1-s_{0}\right)+}\right)\left\|v_{0}\right\|_{H^{1}}^{4}
$$

\section{Proof of estimate (4.5)}

Proof. - The purpose of this section is to prove estimate (4.5), i.e.

$$
\begin{aligned}
& (4.3)=\operatorname{Im} \int_{0}^{\delta} \int_{M} I\left(|u|^{2} u\right)\left(|I u|^{2} \overline{I u}-I\left(|u|^{2} \bar{u}\right)\right) d x d t \lesssim N^{-2+s_{0}+\delta^{\frac{1}{2}}-\left\|v_{0}\right\|_{H^{1}}^{6} .} \\
& \text { томе } 138-2010-\mathrm{N}^{\mathrm{o}} 4
\end{aligned}
$$




$$
\begin{aligned}
(4.3)= & \operatorname{Im} \int_{0}^{\delta} \int_{M} I\left(|u|^{2} u\right)\left(|I u|^{2} \overline{I u}-I\left(|u|^{2} \bar{u}\right)\right) d x d t \\
= & \operatorname{Im} \sum_{N_{0}, \cdots, N_{6}} \int_{0}^{\delta} \int I\left(u^{N_{4}} \bar{u}^{N_{5}} u^{N_{6}}\right)^{N_{0}} \\
& \cdot\left(1-\frac{m_{N}\left(N_{0}^{2}\right)}{m_{N}\left(N_{1}^{2}\right) m_{N}\left(N_{2}^{2}\right) m_{N}\left(N_{3}^{2}\right)}\right) I u^{N_{1}} \overline{I u}^{N_{2}} I u^{N_{3}},
\end{aligned}
$$

where $u^{N_{i}}$ has the same definition as the ones in Section 5. By symmetry argument and because the presence of complex conjugates will play no role here, we can assume $N_{1} \geqslant N_{2} \geqslant N_{3}$, and $N_{4} \geqslant N_{5} \geqslant N_{6}$. As stated in Section 4 , we can assume that $N_{i} \lesssim \sum_{j \in\{0,1,2,3\} \neq i} N_{j}, i=0,1,2,3$, and also $N_{i} \lesssim \sum_{j \in\{0,4,5,6\} \neq i} N_{j}, i=0,4,5,6$.

And if $N_{0}, N_{1}, N_{2}, N_{3} \ll N$, then the right side of (6.1) would be 0 , which is trivial, so we will just deal with the case at least one of them $\gtrsim N$.

Since

$$
\left|1-\frac{m_{N}\left(N_{0}^{2}\right)}{m_{N}\left(N_{1}^{2}\right) m_{N}\left(N_{2}^{2}\right) m_{N}\left(N_{3}^{2}\right)}\right| \lesssim \frac{m_{N}\left(N_{0}^{2}\right)}{m_{N}\left(N_{1}^{2}\right) m_{N}\left(N_{2}^{2}\right) m_{N}\left(N_{3}^{2}\right)}
$$

$$
\begin{aligned}
I(N) \lesssim & \frac{m_{N}\left(N_{0}^{2}\right)}{m_{N}\left(N_{1}^{2}\right) m_{N}\left(N_{2}^{2}\right) m_{N}\left(N_{3}^{2}\right)}\left\|I\left(u^{N_{4}} \bar{u}^{N_{5}} u^{N_{6}}\right)^{N_{0}}\right\|_{L_{t, x}^{2}}\left\|I u^{N_{1}} I u^{N_{2}} I u^{N_{3}}\right\|_{L_{t, x}^{2}} \\
\lesssim & \frac{m_{N}\left(N_{0}^{2}\right)}{m_{N}\left(N_{1}^{2}\right) m_{N}\left(N_{2}^{2}\right) m_{N}\left(N_{3}^{2}\right)}\left\|I u^{N_{1}} I u^{N_{2}} I u^{N_{3}}\right\|_{L_{t, x}^{2}} \\
& \cdot \frac{m_{N}\left(N_{0}^{2}\right)}{m_{N}\left(N_{4}^{2}\right) m_{N}\left(N_{5}^{2}\right) m_{N}\left(N_{6}^{2}\right)}\left\|I u^{N_{4}} I u^{N_{5}} I u^{N_{6}}\right\|_{L_{t, x}^{2}} \\
(6.3)= & (i) \times(i i),
\end{aligned}
$$

where

$$
\text { (i) }=\frac{m_{N}\left(N_{0}^{2}\right)}{m_{N}\left(N_{1}^{2}\right) m_{N}\left(N_{2}^{2}\right) m_{N}\left(N_{3}^{2}\right)}\left\|I u^{N_{1}} I u^{N_{2}} I u^{N_{3}}\right\|_{L_{t, x}^{2}}
$$

and

$$
\text { (ii) }=\frac{m_{N}\left(N_{0}^{2}\right)}{m_{N}\left(N_{4}^{2}\right) m_{N}\left(N_{5}^{2}\right) m_{N}\left(N_{6}^{2}\right)}\left\|I u^{N_{4}} I u^{N_{5}} I u^{N_{6}}\right\|_{L_{t, x}^{2}} .
$$

Let us estimate $(i i)$ first.

Lemma 6.1. - We have

$$
\text { (ii) } \lesssim \begin{cases}N_{4}^{0-} \delta^{\frac{1}{2}-} \prod_{i=4}^{6}\left\|I u^{N_{i}}\right\|_{X_{\delta}^{1, \frac{1}{2}+}} & N_{4} \ll N \\ N^{-1+} N_{4}^{0-} \delta^{\frac{1}{2}-} \prod_{i=4}^{6}\left\|I u^{N_{i}}\right\|_{X_{\delta}^{1, \frac{1}{2}+}} N_{4} \gtrsim N .\end{cases}
$$


Proof. - By Lemma 2.3, Proposition 2.2 and Sobolev embedding theory,

(ii) $\lesssim \frac{m_{N}\left(N_{0}^{2}\right)}{m_{N}\left(N_{4}^{2}\right) m_{N}\left(N_{5}^{2}\right) m_{N}\left(N_{6}^{2}\right)}\left\|I u^{N_{4}} I u^{N_{5}}\right\|_{L_{t, x}^{2}}\left\|I u^{N_{6}}\right\|_{L_{t, x}^{\infty}}$ $\lesssim \frac{m_{N}\left(N_{0}^{2}\right)}{m_{N}\left(N_{4}^{2}\right) m_{N}\left(N_{5}^{2}\right) m_{N}\left(N_{6}^{2}\right)} N_{5}\left\|I u^{N_{4}}\right\|_{X_{\delta}^{0, \frac{1}{4}+}}\left\|I u^{N_{5}}\right\|_{X_{\delta}^{0, \frac{1}{4}+}}\left\|I u^{N_{6}}\right\|_{X_{\delta}^{0, \frac{1}{2}+}}$

$(6.5) \lesssim \frac{m_{N}\left(N_{0}^{2}\right)}{m_{N}\left(N_{4}^{2}\right) m_{N}\left(N_{5}^{2}\right) m_{N}\left(N_{6}^{2}\right)} \frac{1}{N_{4}} \delta^{\frac{1}{2}-} \prod_{i=4}^{6}\left\|I u^{N_{i}}\right\|_{X_{\delta}^{1, \frac{1}{2}+}}$.

(1) $N_{6} \leqslant N_{5} \leqslant N_{4} \ll N$.

$$
\frac{m_{N}\left(N_{0}^{2}\right)}{m_{N}\left(N_{4}^{2}\right) m_{N}\left(N_{5}^{2}\right) m_{N}\left(N_{6}^{2}\right)} \lesssim 1
$$

and

$$
(6.5) \lesssim \frac{1}{N_{4}} \delta^{\frac{1}{2}-} \prod_{i=4}^{6}\left\|I u^{N_{i}}\right\|_{X_{\delta}^{1, \frac{1}{2}+}} \lesssim N_{4}^{0-} \delta^{\frac{1}{2}-} \prod_{i=4}^{6}\left\|I u^{N_{i}}\right\|_{X_{\delta}^{1, \frac{1}{2}+}}
$$

(2) $N_{6} \leqslant N_{5} \ll N \lesssim N_{4}$.

$$
\frac{m_{N}\left(N_{0}^{2}\right)}{m_{N}\left(N_{4}^{2}\right) m_{N}\left(N_{5}^{2}\right) m_{N}\left(N_{6}^{2}\right)} \lesssim N^{-(1-s)} N_{4}^{1-s}
$$

So

$$
\begin{aligned}
(6.5) & \lesssim N^{-(1-s)} N_{4}^{1-s} N_{4}^{-1} \delta^{\frac{1}{2}-} \prod_{i=4}^{6}\left\|I u^{N_{i}}\right\|_{X_{\delta}^{1, \frac{1}{2}+}} \\
& =N^{-(1-s)} N_{4}^{-s} \delta^{\frac{1}{2}-} \prod_{i=4}^{6}\left\|I u^{N_{i}}\right\|_{X_{\delta}^{1, \frac{1}{2}+}} \\
& \lesssim N^{-1+} N_{4}^{0-} \delta^{\frac{1}{2}-} \prod_{i=4}^{6}\left\|I u^{N_{i}}\right\|_{X_{\delta}^{1, \frac{1}{2}+} \cdot}
\end{aligned}
$$

(3) $N_{6} \ll N \lesssim N_{5} \leqslant N_{4}$.

Then

$$
\frac{m_{N}\left(N_{0}^{2}\right)}{m_{N}\left(N_{4}^{2}\right) m_{N}\left(N_{5}^{2}\right) m_{N}\left(N_{6}^{2}\right)} \lesssim N^{-2(1-s)}\left(N_{4} N_{5}\right)^{1-s},
$$

TOME $138-2010-\mathrm{N}^{\circ} 4$ 
and

$$
\begin{aligned}
(6.5) & \lesssim N^{-2(1-s)}\left(N_{4} N_{5}\right)^{1-s} N_{4}^{-1} \delta^{\frac{1}{2}-} \prod_{i=4}^{6}\left\|I u^{N_{i}}\right\|_{X_{\delta}^{1, \frac{1}{2}+}} \\
& \lesssim N^{-2(1-s)} N_{4}^{-s} N_{5}^{1-s} \delta^{\frac{1}{2}-} \prod_{i=4}^{6}\left\|I u^{N_{i}}\right\|_{X_{\delta}^{1, \frac{1}{2}+}} \\
& \lesssim N^{-1+} N_{4}^{0-} \delta^{\frac{1}{2}-} \prod_{i=4}^{6}\left\|I u^{N_{i}}\right\|_{X_{\delta}^{1, \frac{1}{2}+}}
\end{aligned}
$$

with $s>\frac{1}{2}$.

(4) $N \lesssim N_{6} \leqslant N_{5} \leqslant N_{4}$.

Then

$$
\frac{m_{N}\left(N_{0}^{2}\right)}{m_{N}\left(N_{4}^{2}\right) m_{N}\left(N_{5}^{2}\right) m_{N}\left(N_{6}^{2}\right)} \lesssim N^{-3(1-s)}\left(N_{4} N_{5} N_{6}\right)^{1-s},
$$

and do the same thing as (3), we have

$$
(6.5) \lesssim N^{-1+} N_{4}^{0-} \delta^{\frac{1}{2}-} \prod_{i=4}^{6}\left\|I u^{N_{i}}\right\|_{X_{\delta}^{1, \frac{1}{2}+}},
$$

with the assumption that $s>\frac{2}{3}$.

Hence, conclusively,

$$
\text { (ii) } \lesssim N_{4}^{0-} \delta^{\frac{1}{2}-} \prod_{i=4}^{6}\left\|I u^{N_{i}}\right\|_{X_{\delta}^{1, \frac{1}{2}+}} .
$$

Then for (i), we can deal with it in the same cases as (ii). As at least $N_{1} \gtrsim N$, we just need to consider about the case (2), (3), (4), which gives

$$
\text { (i) } \lesssim N^{-1+} N_{1}^{0-} \delta^{\frac{1}{2}-} \prod_{i=1}^{3}\left\|I u^{N_{i}}\right\|_{X_{\delta}^{1, \frac{1}{2}+}} \cdot
$$

Therefore, when $N_{4} \gtrsim N$, from (6.3), (6.4) and (6.10),

$$
I(N) \lesssim N_{1}^{0-} N_{4}^{0-} N^{-2+} \delta^{1-} \prod_{i=1}^{6}\left\|I u^{N_{i}}\right\|_{X_{\delta}^{1, \frac{1}{2}+}},
$$

and

$$
\sum_{N_{0} \cdots N_{6}} I(N) \lesssim N^{-2+} \delta^{1-}\|I u\|_{X_{\delta}^{1, \frac{1}{2}+}}^{6} \lesssim N^{-2+} \delta^{1-}\left\|v_{0}\right\|_{X_{\delta}^{1, \frac{1}{2}+}}^{6} \lesssim N^{-2+s_{0}+} \delta^{\frac{1}{2}-}\left\|v_{0}\right\|_{H^{1}}^{6} .
$$

For $N_{4} \ll N$, which means that $N_{0} \lesssim N_{4} \ll N$, so $N_{1} \geqslant N_{2} \gtrsim N$, and

$$
\frac{m_{N}\left(N_{0}^{2}\right)}{m_{N}\left(N_{1}^{2}\right) m_{N}\left(N_{2}^{2}\right) m_{N}\left(N_{3}^{2}\right)} \lesssim N^{-2(1-s)}\left(N_{1} N_{2}\right)^{1-s} \frac{1}{m_{N}\left(N_{3}^{2}\right)} .
$$




\section{Hence}

(i) $\lesssim N^{-2(1-s)}\left(N_{1} N_{2}\right)^{1-s} \frac{1}{m_{N}\left(N_{3}^{2}\right)}\left\|I u^{N_{1}} I u^{N_{2}}\right\|_{L_{t, x}^{2}}\left\|I u^{N_{3}}\right\|_{L_{t}^{\infty} H_{x}^{1}}$

$$
\lesssim N^{-2(1-s)}\left(N_{1} N_{2}\right)^{1-s} \frac{1}{m_{N}\left(N_{3}^{2}\right)} N_{2}^{s_{0}+}\left\|I u^{N_{1}}\right\|_{X_{\delta}^{0, \frac{1}{2}+}}\left\|I u^{N_{2}}\right\|_{X_{\delta}^{0, \frac{1}{2}+}}\left\|I u^{N_{3}}\right\|_{X_{\delta}^{1, \frac{1}{2}+}}
$$

$$
\lesssim N^{-2(1-s)} \frac{1}{m_{N}\left(N_{3}^{2}\right)} N_{1}^{-s} N_{2}^{-s+s_{0}+} \prod_{i=1}^{3}\left\|I u^{N_{i}}\right\|_{X_{\delta}^{1, \frac{1}{2}+}} .
$$

Discussing for $N_{3} \ll N$ or $N_{3} \gtrsim N$, it is easy to get

$$
(6.13) \lesssim N^{-2+s_{0}+} N_{1}^{0-} \prod_{i=1}^{3}\left\|I u^{N_{i}}\right\|_{X_{\delta}^{1, \frac{1}{2}+}} .
$$

Therefore, for this case,

$$
\sum I(N) \lesssim N^{-2+s_{0}+} \delta^{\frac{1}{2}-}\|I u\|_{X_{\delta}^{1, \frac{1}{2}+}}^{6} \lesssim N^{-2+s_{0}+} \delta^{\frac{1}{2}-}\left\|v_{0}\right\|_{H^{1}}^{6} .
$$

\section{Proof of Theorem 1.2}

Proof. - By Proposition 4.1, (2.6) and (2.7), we have,

$E(v(\delta))-E\left(v_{0}\right) \lesssim\left(N^{-\left(1-s_{0}\right)+} \delta^{\frac{1}{2}-}+N^{-2\left(1-s_{0}\right)+}\right)\left\|v_{0}\right\|_{H^{1}}^{4}+N^{-2+s_{0}+} \delta^{\frac{1}{2}-}\left\|v_{0}\right\|_{H^{1}}^{6}$

$$
\lesssim\left(N^{-\left(1-s_{0}\right)+} \delta^{\frac{1}{2}-}+N^{-2\left(1-s_{0}\right)+}\right) N^{4(1-s)}+N^{-2+s_{0}+} \delta^{\frac{1}{2}-} N^{6(1-s)}
$$

$$
\lesssim N^{3-4 s+s_{0}+} \delta^{\frac{1}{2}-}+N^{2-4 s+2 s_{0}+}+N^{4-6 s+s_{0}+} \delta^{\frac{1}{2}-} \text {. }
$$

By the definition of energy, and Gagliardo-Nirenberg inequality, we have

$$
E\left(v_{0}\right)=\frac{1}{2}\left\|\nabla v_{0}\right\|_{L_{x}^{2}}^{2}+\frac{1}{4}\left\|v_{0}\right\|_{L_{x}^{4}}^{4} \leqslant \frac{1}{2}\left\|\nabla v_{0}\right\|_{L_{x}^{2}}^{2}+C\left\|v_{0}\right\|_{L_{x}^{2}}^{2}\left\|\nabla v_{0}\right\|_{L_{x}^{2}}^{2} \leqslant C\left\|v_{0}\right\|_{H_{x}^{1}}^{2} \lesssim N^{2(1-s)} .
$$

Hence, for any given time $T \gg 1$, the number of iterate is $\frac{T}{\delta}$ with $\delta \gtrsim$ $N^{-\frac{2\left(1-s_{0}\right)(1-s)}{s-s_{0}}-}$, and the total energy is

$$
\begin{aligned}
E(v(T)) \lesssim & E(v(0))+\frac{T}{\delta}\left(N^{3-4 s+s_{0}+} \delta^{\frac{1}{2}-}+N^{2-4 s+2 s_{0}+}+N^{4-6 s+s_{0}+} \delta^{\frac{1}{2}-}\right) \\
\lesssim & N^{2(1-s)}+T\left(N^{3-4 s+s_{0}+} \delta^{-\frac{1}{2}-}+N^{2-4 s+2 s_{0}+} \delta^{-1}+N^{4-6 s+s_{0}+} \delta^{-\frac{1}{2}-}\right) \\
(7.3) \lesssim & N^{2(1-s)}+T\left(N^{3-4 s+s_{0}+\frac{(1-s)\left(1-s_{0}\right)}{s-s_{0}}+}\right. \\
& \left.+N^{2-4 s+2 s_{0}+\frac{2(1-s)\left(1-s_{0}\right)}{s-s_{0}}+}+N^{4-6 s+s_{0}+\frac{(1-s)\left(1-s_{0}\right)}{s-s_{0}}+}\right) .
\end{aligned}
$$

Now we should take some proper $N=N(T)$, such that $T\left(N^{3-4 s+s_{0}+\frac{(1-s)\left(1-s_{0}\right)}{s-s_{0}}+}+N^{2-4 s+2 s_{0}+\frac{2(1-s)\left(1-s_{0}\right)}{s-s_{0}}+}+N^{4-6 s+s_{0}+\frac{(1-s)\left(1-s_{0}\right)}{s-s_{0}}+}\right) \lesssim N^{2(1-s)}$, 
so that

$$
\|u(T)\|_{H_{x}^{s}} \lesssim\|I u(T)\|_{H_{x}^{1}}=\|v(T)\|_{H_{x}^{1}} \lesssim \sqrt{E(v(T))}+\|v(T)\|_{L_{x}^{2}} \lesssim N^{1-s}=T^{\alpha},
$$

with $\alpha>0$. Here, we use the conservation of $L^{2}$-mass of $u(t)$, which gives

$$
\|v(T)\|_{L_{x}^{2}}=\left\|I_{N} u(T)\right\|_{L_{x}^{2}} \leqslant\|u(T)\|_{L_{x}^{2}}=\left\|u_{0}\right\|_{L_{x}^{2}} .
$$

Since

$$
3-4 s+s_{0}+\frac{(1-s)\left(1-s_{0}\right)}{s-s_{0}}+>2-4 s+2 s_{0}+\frac{2(1-s)\left(1-s_{0}\right)}{s-s_{0}}+
$$

for $s>\frac{1+s_{0}}{2}$, and

$$
3-4 s+s_{0}+\frac{(1-s)\left(1-s_{0}\right)}{s-s_{0}}+>4-6 s+s_{0}+\frac{(1-s)\left(1-s_{0}\right)}{s-s_{0}}+
$$

for $s>\frac{1}{2}$,

$$
\begin{aligned}
& T\left(N^{3-4 s+s_{0}+\frac{(1-s)\left(1-s_{0}\right)}{s-s_{0}}+}+N^{2-4 s+2 s_{0}+\frac{2(1-s)\left(1-s_{0}\right)}{s-s_{0}}+}\right. \\
& \left.\quad+N^{4-6 s+s_{0}+\frac{(1-s)\left(1-s_{0}\right)}{s-s_{0}}+}\right) \lesssim T N^{3-4 s+s_{0}+\frac{(1-s)\left(1-s_{0}\right)}{s-s_{0}}+} \lesssim N^{2(1-s)}
\end{aligned}
$$

by taking $N \sim T^{\frac{s-s_{0}}{2 s^{2}-4 s_{0} s+s_{0}^{2}+2 s_{0}-1}}+$, for $s>\frac{\sqrt{2}}{2}+\left(1-\frac{\sqrt{2}}{2}\right) s_{0}>\max \left\{\frac{1+s_{0}}{2}, \frac{2}{3}\right\}$.

Therefore, by (7.4)

$$
\|u(T)\|_{H^{s}} \lesssim T^{\frac{\left(s-s_{0}\right)(1-s)}{2 s^{2}-4 s_{0} s+s_{0}^{2}+2 s_{0}-1}}+.
$$

\section{Appendix}

In this section, we will prove Lemma 3.2 and Lemma 5.1.

\subsection{Proof of Lemma 3.2}

Proof. - It is just a little modification of Proposition 2.5 of [10].

Assume by density that $u \in C_{0}^{\infty}(\mathbb{R} \times M)$. By duality argument, we just need to prove

$$
\left|\int_{\mathbb{R} \times M} \bar{\varphi} I_{N}\left(|u|^{2} u\right) d x d t\right| \leqslant C\|\varphi\|_{X^{-1, b^{\prime}(s-)}}\left\|I_{N} u\right\|_{X^{1, b^{\prime}(s-)}}^{3},
$$

for $\varphi \in X^{-1, b^{\prime}(s-)}$. Denote

$$
\begin{gathered}
\varphi^{N_{0}}=\sum_{N_{0} \leqslant<\mu_{k}>\frac{1}{2}<2 N_{0}} P_{k} \varphi, \\
u^{N_{j}=} P_{k} u, \text { for } j=1,2,3,
\end{gathered}
$$

BULletin DE LA SOCiÉtÉ MATHÉMATIQUE DE FRANCE 
and

then

$$
I(N)=\left|\int_{\mathbb{R} \times M} \bar{\varphi}^{N_{0}} m\left(N_{0}\right) u^{N_{1}} \bar{u}^{N_{2}} u^{N_{3}} d x d t\right|,
$$

$$
\left|\int_{\mathbb{R} \times M} \bar{\varphi} I_{N}\left(|u|^{2} u\right) d x d t\right| \lesssim \sum_{N_{0}, N_{1}, N_{2}, N_{3}} I(N) .
$$

By symmetry argument and because the presence of complex conjugates will play no role here, we can assume $N_{1} \geqslant N_{2} \geqslant N_{3}$. Also by Lemma 2.6 of [10], the condition that $s<1$, which gives $\frac{1}{4}<b^{\prime}(s-)$, we can manage the case $N_{0} \geqslant C\left(N_{1}+N_{2}+N_{3}\right)$ by some similar argument as in section 5.1 .2 , so we just need to estimate the case $N_{0} \lesssim N_{1}+N_{2}+N_{3}$, which implies $N_{0} \lesssim N_{1}$.

Therefore,

$$
\begin{aligned}
I(N) & \lesssim \frac{m_{N}\left(N_{0}^{2}\right)}{m_{N}\left(N_{1}^{2}\right) m_{N}\left(N_{2}^{2}\right) m_{N}\left(N_{3}^{2}\right)} \int \bar{\varphi}^{N_{0}} I_{N} u^{N_{1}}{\overline{I_{N} u}}^{N_{2}} I_{N} u^{N_{3}} \\
& \lesssim \frac{m_{N}\left(N_{0}^{2}\right)}{m_{N}\left(N_{1}^{2}\right) m_{N}\left(N_{2}^{2}\right) m_{N}\left(N_{3}^{2}\right)}\left\|\varphi^{N_{0}} I_{N} u^{N_{2}}\right\|_{L_{t, x}^{2}}\left\|I_{N} u^{N_{1}} I_{N} u^{N_{3}}\right\|_{L_{t, x}^{2}} \\
& \lesssim \frac{m_{N}\left(N_{0}^{2}\right)}{m_{N}\left(N_{1}^{2}\right) m_{N}\left(N_{2}^{2}\right) m_{N}\left(N_{3}^{2}\right)}\left(N_{2} N_{3}\right)^{s-}\left\|\varphi^{N_{0}}\right\|_{X^{0, b^{\prime}(s-)}} \prod_{i=1}^{3}\left\|I_{N} u\right\|_{X^{0, b^{\prime}(s-)}}
\end{aligned}
$$

$$
\begin{aligned}
& \lesssim \frac{m_{N}\left(N_{0}^{2}\right)}{m_{N}\left(N_{1}^{2}\right) m_{N}\left(N_{2}^{2}\right) m_{N}\left(N_{3}^{2}\right)}\left(\frac{N_{0}}{N_{1}}\right)\left(N_{2} N_{3}\right)^{s-1-} \\
& \cdot\left\|\varphi^{N_{0}}\right\|_{X^{-1, b^{\prime}(s-)}} \prod_{i=1}^{3}\left\|I_{N} u\right\|_{X^{1, b^{\prime}(s-)}},
\end{aligned}
$$

by Lemma 2.3.

If $N_{i} \ll N$

$$
\frac{N_{i}^{s-1-}}{m_{N}\left(N_{i}^{2}\right)}=N_{i}^{s-1-} .
$$

If $N_{i} \gtrsim N$

$$
\frac{N_{i}^{s-1-}}{m_{N}\left(N_{i}^{2}\right)}=\left(\frac{N_{i}}{N}\right)^{1-s} N_{i}^{s-1-}=N^{s-1} N_{i}^{0-},
$$

with $s_{0}<s<1$.

Therefore, (8.3) turns to be

$$
\begin{aligned}
& \sum_{N_{0} \lesssim\left(N_{1}+N_{2}+N_{3}\right)} I(N) \\
\lesssim & \sum_{N_{0} \lesssim N_{1}} \frac{m_{N}\left(N_{0}^{2}\right)}{m_{N}\left(N_{1}^{2}\right)}\left(\frac{N_{0}}{N_{1}}\right)\left\|\varphi^{N_{0}}\right\|_{X^{-1, b^{\prime}(s-)}}\left\|I_{N} u^{N_{1}}\right\|_{X^{1, b^{\prime}(s-)}}\left\|I_{N} u\right\|_{X^{1, b^{\prime}(s-)}}^{2} .
\end{aligned}
$$


Case 1. $N_{0} \lesssim N_{1} \ll N: \frac{m_{N}\left(N_{0}^{2}\right)}{m_{N}\left(N_{1}^{2}\right)}\left(\frac{N_{0}}{N_{1}}\right) \sim\left(\frac{N_{0}}{N_{1}}\right) \lesssim\left(\frac{N_{0}}{N_{1}}\right)^{s}$.

Case $2 . N_{0} \ll N \lesssim N_{1}: \frac{m_{N}\left(N_{0}^{2}\right)}{m_{N}\left(N_{1}^{2}\right)}\left(\frac{N_{0}}{N_{1}}\right) \lesssim\left(\frac{N_{1}}{N}\right)^{1-s}\left(\frac{N_{0}}{N_{1}}\right) \lesssim\left(\frac{N_{0}}{N_{1}}\right)^{s}$.

Case 3. $N \lesssim N_{0} \lesssim N_{1}: \frac{m_{N}\left(N_{0}^{2}\right)}{m_{N}\left(N_{1}^{2}\right)}\left(\frac{N_{0}}{N_{1}}\right) \lesssim\left(\frac{N_{1}}{N_{0}}\right)^{1-s}\left(\frac{N_{0}}{N_{1}}\right)=\left(\frac{N_{0}}{N_{1}}\right)^{s}$.

Hence,

$\sum_{N_{0}, N_{1}, N_{2}, N_{3}} I(N) \lesssim \sum_{N_{0} \lesssim N_{1}}\left(\frac{N_{0}}{N_{1}}\right)^{s}\left\|\varphi^{N_{0}}\right\|_{X^{-1, b^{\prime}(s-)}}\left\|I_{N} u^{N_{1}}\right\|_{X^{1, b^{\prime}(s-)}}\left\|I_{N} u\right\|_{X^{1, b^{\prime}(s-)}}^{2}$.

Then, because for $N_{0} \lesssim N_{1}$, there is some fixed positive integer $l_{0}$ such that $N_{1}=2^{l} N_{0}$, where $l \geqslant-l_{0}$, it has

$$
\begin{aligned}
\sum_{N_{0} \lesssim N_{1}}\left(\frac{N_{0}}{N_{1}}\right)^{s} & \left\|\varphi^{N_{0}}\right\|_{X^{-1, b^{\prime}}(s-)}\left\|I_{N} u^{N_{1}}\right\|_{X^{1, b^{\prime}}(s-)} \\
& =\sum_{l \geqslant-l_{0}} \sum_{N_{0}} 2^{-s l}\left\|\varphi^{N_{0}}\right\|_{X^{-1, b^{\prime}(s-)}}\left\|I_{N} u^{2^{l} N_{0}}\right\|_{X^{1, b^{\prime}(s-)}} \\
& \lesssim \sum_{l \geqslant-l_{0}} 2^{-s l}\left(\sum_{N_{0}}\left\|\varphi^{N_{0}}\right\|_{X^{-1, b^{\prime}(s-)}}^{2}\right)^{\frac{1}{2}}\left(\sum_{N_{0}}\left\|I_{N} u^{2^{l} N_{0}}\right\|_{X^{1, b^{\prime}(s-)}}^{2}\right)^{\frac{1}{2}} \\
& \lesssim\|\varphi\|_{X^{-1, b^{\prime}(s-)}}\left\|I_{N} u\right\|_{X^{1, b^{\prime}(s-)}},
\end{aligned}
$$

which ends the proof for this lemma.

\subsection{Proof of Lemma 5.1}

Proof. - Let

$$
1=\theta_{0}(\xi)+\sum_{j=1}^{\infty} \theta\left(2^{-j} \xi\right)
$$

be a dyadic partition of unity in $\mathbb{R}^{2}, \theta_{0} \in C_{0}^{\infty}\left(\mathbb{R}^{2}\right), \theta \in C_{0}^{\infty}\left(\mathbb{R}^{2} \backslash\{0\}\right)$, and $\operatorname{supp}(\theta) \subset(1,+\infty)$.

So

$$
\begin{aligned}
m(\xi) & =\theta_{0}(\xi) m(\xi)+\sum_{j=1}^{\infty} \theta\left(2^{-j} \xi\right)|\xi|^{-\frac{1-s}{2}} \\
& =\theta_{0}(\xi) m(\xi)+\sum_{j=1}^{\infty} 2^{-\frac{j}{2}(1-s)} \theta\left(2^{-j} \xi\right)\left|2^{-j} \xi\right|^{-\frac{1-s}{2}}
\end{aligned}
$$


which gives

$$
\begin{aligned}
& m\left(N^{-2} \triangle\right)=\theta_{0}\left(N^{-2} \triangle\right) m\left(N^{-2} \triangle\right)+\sum_{j=1}^{\infty} 2^{-\frac{j}{2}(1-s)} \theta\left(2^{-j} N^{-2} \triangle\right)\left|2^{-j} N^{-2} \triangle\right|^{-\frac{1-s}{2}} \\
& (8.8) \quad=\sum_{j=0}^{\infty} 2^{-\frac{j}{2}(1-s)} \varphi_{1 j}\left(h_{j}^{2} \triangle\right),
\end{aligned}
$$

here $h_{j}=2^{-\frac{j}{2}} N^{-1}(j \geqslant 0)$, and

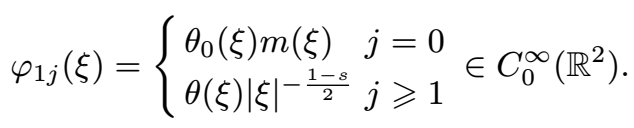

By Proposition 2.1 of [9], there is a sequence $\left\{\psi_{1 i j}(x, \xi)\right\}$ of $C_{0}^{\infty}\left(M \times \mathbb{R}^{d}\right)$, and $\chi_{3}$, which is equal to 1 near the support of $\chi_{2}$ and $\operatorname{supp}\left(\chi_{3}\right)$ is a slightly larger than $\operatorname{supp}\left(\chi_{2}\right)$, such that for every $K \in \mathbb{N}, f \in C^{\infty}(M)$

$$
\chi_{2} \varphi_{1 j}\left(h_{j}^{2} \triangle\right) f-\sum_{i=0}^{K-1} h_{j}^{i} \psi_{1 i j}\left(x, h_{j} D\right) \chi_{3} f=R_{1 j}\left(x, h_{j} D, h_{j}\right) f
$$

here $\psi_{10}=\chi_{2}(x) \varphi_{1 j}\left(p_{2}(x, \xi)\right)$, and for $i \geqslant 1, \psi_{1 i j}=\sum_{k \geqslant 2} \frac{\varphi_{1}^{(k-1)}\left(p_{2}\right)}{(k-1) !} q_{1 i j k}$, $q_{1 i j k}(x, \xi)$ are polynomials in $\xi$ of degree less or equal to $2(k-1)$ and supported in the set $\left\{x \in \operatorname{supp}\left(\chi_{3}\right)\right\}, p_{2}$ is the principal symbol of $\triangle_{g}, a|\xi|^{2} \leqslant p_{2}(x, \xi) \leqslant$ $b|\xi|^{2}$, and

$$
\left\|R_{1 j} f\right\|_{L_{x}^{2}} \lesssim h_{j}^{K}\|f\|_{L_{x}^{2}}
$$

Let

$$
m_{1}\left(x, N^{-1} D\right)=\sum_{j=0}^{\infty} \sum_{i=0}^{K-1} 2^{-\frac{j}{2}(1-s)} h_{j}^{i} \psi_{1 i j}\left(x, h_{j} D\right) .
$$

Then, similarly, for $m^{-1}\left(N^{-2} \triangle\right)$,

$$
m^{-1}\left(N^{-2} \triangle\right)=\sum_{j=0}^{\infty} 2^{\frac{j}{2}(1-s)} \varphi_{2 j}\left(h_{j}^{2} \triangle\right),
$$

here

$$
\varphi_{2 j}(\xi)=\left\{\begin{array}{l}
\theta_{0}(\xi) m^{-1}(\xi) \\
\theta(\xi)|\xi|^{\frac{1-s}{2}} \quad j \geqslant 1
\end{array} \in C_{0}^{\infty}\left(\mathbb{R}^{2}\right) .\right.
$$

Also there are $\left\{\psi_{2 i j}(x, \xi)\right\}$ and $R_{2 j}\left(x, h_{j} D, h_{j}\right)$ such that

$$
\chi_{2} \varphi_{2 j}\left(h_{j}^{2} \triangle\right) f-\sum_{i=0}^{K-1} h_{j}^{i} \psi_{2 i j}\left(x, h_{j} D\right) \chi_{3} f=R_{2 j}\left(x, h_{j} D, h_{j}\right) f,
$$


and

$$
\left\|R_{2 j} f\right\|_{L_{x}^{2}} \lesssim h_{j}^{K}\|f\|_{L_{x}^{2}}
$$

Denote

$$
m_{2}\left(x, N^{-1} D\right)=\sum_{j=0}^{\infty} \sum_{i=0}^{K-1} 2^{\frac{j}{2}(1-s)} h_{j}^{i} \psi_{2 i j}\left(x, h_{j} D\right)
$$

Now, let us deal with

$$
\left|\int_{0}^{\delta} \int \nabla \overline{I u} \chi_{2}[I, g] \chi_{2} I^{-1} \nabla I u\right|=\left|\int_{0}^{\delta} \int \bar{h} \chi_{2}\left[m\left(N^{-2} \triangle\right), g\right] m^{-1}\left(N^{-2} \triangle\right) h\right|,
$$

here $h=\nabla I u$.

By the properties of $\chi_{3}$, we obtain

$$
\begin{aligned}
\int_{0}^{\delta} \int & \bar{h} \chi_{2}\left(\left[m\left(N^{-2} \triangle\right), g\right] m^{-1}\left(N^{-2} \triangle\right)\right) h \\
= & \int_{0}^{\delta} \int \bar{h}\left[\chi_{2} m\left(N^{-2} \triangle\right)-m_{1}\left(x, N^{-1} D\right) \chi_{3}, g\right] \tilde{\chi}_{2} m^{-1}\left(N^{-2} \triangle\right) h \\
& +\int_{0}^{\delta} \int \bar{h}\left[m_{1}\left(x, N^{-1} D\right) \chi_{3}, g\right] \tilde{\chi}_{2} m^{-1}\left(N^{-2} \triangle\right) h \\
= & \int_{0}^{\delta} \int \bar{h}\left[\chi_{2} m\left(N^{-2} \triangle\right)-m_{1}\left(x, N^{-1} D\right) \chi_{3}, g\right]\left(\tilde{\chi}_{2} m^{-1}\left(N^{-2} \triangle\right)-m_{2}\left(x, N^{-1} D\right) \chi_{3}\right) h \\
& +\int_{0}^{\delta} \int \bar{h}\left[m_{1}\left(x, N^{-1} D\right) \chi_{3}, g\right]\left(\tilde{\chi}_{2} m^{-1}\left(N^{-2} \triangle\right)-m_{2}\left(x, N^{-1} D\right) \chi_{3}\right) h \\
& +\int_{0}^{\delta} \int \bar{h}\left[\chi_{2} m\left(N^{-2} \triangle\right)-m_{1}\left(x, N^{-1} D\right) \chi_{3}, g\right] m_{2}\left(x, N^{-1} D\right) \chi_{3} h \\
& +\int_{0}^{\delta} \int \bar{h}\left[m_{1}\left(x, N^{-1} D\right) \chi_{3}, g\right] m_{2}\left(x, N^{-1} D\right) \chi_{3} h \\
(8.16) & (\mathrm{i})+(\mathrm{ii})+(\mathrm{iii})+(\mathrm{iv}),
\end{aligned}
$$

with $\tilde{\chi}_{2}$ equal to 1 near the support of $\chi_{2}$, and $\operatorname{supp}\left(\tilde{\chi}_{2}\right)$ is a little larger than $\operatorname{supp}\left(\chi_{2}\right)$. 
8.2.1. Study of (i), (ii) and (iii). - For (i), (ii), (iii), we will use the same method, and take (iii) for example.

(iii) $\lesssim\|h\|_{L_{t, x}^{2}}\left\|\left[\chi_{2} m\left(N^{-2} \triangle\right)-m_{1}\left(x, N^{-1} D\right) \chi_{3}, g\right] m_{2}\left(x, N^{-1} D\right) \chi_{3} h\right\|_{L_{t, x}^{2}}$

$$
\lesssim \sum_{j, k \geqslant 0} \sum_{i=0}^{K-1} 2^{\frac{k-j}{2}(1-s)} h_{k}^{i}\|h\|_{L_{t, x}^{2}}\left\|\left[R_{1 j}\left(x, h_{j} D, h_{j}\right), g\right] \psi_{2 i k}\left(x, h_{k} D\right) h\right\|_{L_{t, x}^{2}}
$$

$$
\lesssim \sum_{j, k \geqslant 0} \sum_{i=0}^{K-1} 2^{\frac{k-j}{2}(1-s)} h_{k}^{i}\|h\|_{L_{t, x}^{2}}\left(\left\|R_{1 j}\left(g \psi_{2 i k} h\right)\right\|_{L_{t, x}^{2}}+\left\|g\left(R_{1 j} \psi_{2 i k} h\right)\right\|_{L_{t . x}^{2}}\right) .
$$

$$
\begin{gathered}
\left\|\partial_{x}^{\alpha} g\right\|_{L_{x}^{\infty}}=\left\|\partial_{x}^{\alpha}\left(\chi_{1}\left(N^{-2+} \triangle\right) u \overline{\chi_{1}\left(N^{-2+} \triangle\right) u}\right)\right\|_{L_{x}^{\infty}} \\
\lesssim N^{|\alpha|-}\left\|\chi_{1}\left(N^{-2+} \triangle\right) u\right\|_{H_{x}^{1}}^{2} \lesssim N^{|\alpha|-}\|I u\|_{H_{x}^{1}}^{2}, \text { for }|\alpha| \geqslant 1 \\
\|g\|_{L_{x}^{\infty}} \lesssim N^{0+}\|I u\|_{H^{1}}^{2} \\
\left|\partial_{x}^{\alpha} \psi_{2 i k}\left(x, h_{k} \xi\right)\right| \lesssim h_{k}^{1-s}|\xi|^{1-s} \lesssim 1
\end{gathered}
$$

and

$$
h_{k}^{|\alpha|} N^{|\alpha|-}=2^{-\frac{k}{2}|\alpha|} N^{0-},
$$

so by the classical pseudodifferential estimates, we just need to consider $|k-j| \leqslant$ $\nu$, here $\nu$ is a fixed positive constant.

$$
\begin{aligned}
\sum_{j} & \sum_{|k-j| \leqslant \nu} \sum_{i=0}^{K-1} 2^{\frac{k-j}{2}(1-s)} h_{k}^{i}\|h\|_{L_{t, x}^{2}}\left(\left\|R_{1 j}\left(g \psi_{2 i k} h\right)\right\|_{L_{t, x}^{2}}+\left\|g\left(R_{1 j} \psi_{2 i k} h\right)\right\|_{L_{t, x}^{2}}\right) \\
& \lesssim \sum_{j} \sum_{|k-j| \leqslant \nu} \sum_{i=0}^{K-1} 2^{\frac{k-j}{2}(1-s)} h_{k}^{i}\|h\|_{L_{t, x}^{2}} h_{j}^{K}\|g\|_{L_{t, x}^{\infty}}\|h\|_{L_{t, x}^{2}} \\
& \lesssim \sum_{j} \sum_{|k-j| \leqslant \nu} \sum_{i=0}^{K-1} 2^{\frac{k-j}{2}(1-s)} h_{k}^{i} h_{j}^{K}\|h\|_{X_{\delta}^{0,0}}^{2} N^{0+}\|I u\|_{L_{t}^{\infty} H_{x}^{1}}^{2} \\
& \lesssim \sum_{j} \sum_{|k-j| \leqslant \nu} \sum_{i=0}^{K-1} 2^{\frac{k-j}{2}(1-s)} h_{k}^{i} h_{j}^{K} N^{0+} \delta^{1-}\|I u\|_{X_{\delta}^{1, \frac{1}{2}+}}^{4} \\
& \lesssim N^{-K+} \delta^{1-}\|I u\|_{X_{\delta}^{1, \frac{1}{2}+} \cdot}^{4}
\end{aligned}
$$

Hence by taking $K=2$, the estimate for $(i i i)$ meets the requirement of the lemma. And for $(i)$ and $(i i)$, the results are the same or even better. 
8.2.2. Deal with (iv). — Now, let us estimate (iv).

$$
\begin{aligned}
(\text { iv })= & \iint \bar{h}\left[m_{1}\left(x, N^{-1} D\right) \chi_{3}, g\right] m_{2}\left(x, N^{-1} D\right) \chi_{3} h \\
= & \sum_{j, k \geqslant 0} \sum_{i, l=0}^{K-1} 2^{\frac{k-j}{2}(1-s) \iint \bar{h}\left[\psi_{1 i j}\left(x, h_{j} D\right), g\right] \psi_{2 l k}\left(x, h_{k} D\right) \tilde{\chi}_{3} h} \\
= & \sum_{N_{0}, N_{1}, N_{2}, N_{3}} \sum_{j, k \geqslant 0} \sum_{i, l=0}^{K-1} 2^{\frac{k-j}{2}(1-s)} \\
& \cdot \iint \bar{h}^{N_{0}}\left[\psi_{1 i j}\left(x, h_{j} D\right), \chi_{1}\left(N^{-2+} \triangle\right) u^{N_{1}} \overline{\chi_{1}\left(N^{-2+} \triangle\right) u^{N_{2}}}\right] \\
& \psi_{2 l k}\left(x, h_{k} D\right) \tilde{\chi}_{3} h^{N_{3}},
\end{aligned}
$$

where $\tilde{\chi}_{3} \in C_{0}^{\infty}$, which equals 1 near support $\chi_{3}, h^{N_{i}}, u^{N_{i}}$ is defined as above. Denote $\tilde{g}=\chi_{1}\left(N^{-2+} \triangle\right) u^{N_{1}} \overline{\chi_{1}\left(N^{-2+} \triangle\right) u^{N_{2}}}$.

First, for the same reason as above, we just need to estimate the case $|k-j| \leqslant \nu, N_{1}, N_{2} \lesssim N^{1-}$, and $N_{0} \sim N_{3} \gtrsim N$, otherwise, $\left[\psi_{1 i j}\left(x, h_{j} D\right), \chi_{1}\left(N^{-2+} \triangle\right) u^{N_{1}} \overline{\chi_{1}\left(N^{-2+} \triangle\right) u^{N_{2}}}\right]=0$. [20].

By (8.18), the following lemma is from Theorem 2.6.5 and Remark 2.6.7 of

LEMma 8.1. - We have

$$
\left[\psi_{1 i j}, \tilde{g}\right]=\sum_{|\alpha|=1}^{K^{\prime}-1} \frac{h_{j}^{|\alpha|}}{i^{|\alpha|}|\alpha| !} \partial_{\xi}^{\alpha} \psi_{1 i j} \partial_{x}^{\alpha} \tilde{g}+R_{i j}\left(x, h_{j} \xi, h_{j}\right),
$$

such that

$$
\left\|R_{i j}\right\|_{L_{x}^{2} \rightarrow L_{x}^{2}} \lesssim h_{j}^{K^{\prime}} N^{K^{\prime}(1-\epsilon)}\left(N_{1} N_{2}\right)^{0-}\|I u\|_{H^{1}}^{2},
$$

where $\epsilon>0$ depends on the eigenvalue scale of $\chi_{1}\left(N^{-2+} \triangle\right) u$.

So take $K^{\prime}$ large enough such that $K^{\prime} \epsilon>1-s_{0}$, and do the same thing as (iii), then we can get the result what we want for $R_{i}$. Therefore, the left terms are $\sum_{|\alpha|=1}^{K^{\prime}-1} \frac{h_{j}^{|\alpha|}}{i^{|\alpha|}|\alpha| !} \partial_{\xi}^{\alpha} \psi_{1 i j} \partial_{x}^{\alpha} \tilde{g}$, and what we will estimate is

$$
\begin{aligned}
\sum_{N_{0}, N_{1}, N_{2}, N_{3}} \sum_{j} \sum_{|k-j| \leqslant \nu} & \sum_{i, l=0}^{K-1} \sum_{|\alpha|=1}^{K^{\prime}-1} 2^{\frac{k-j}{2}(1-s)} \frac{h_{j}^{|\alpha|}}{i^{|\alpha|} \alpha !} \\
& \cdot \iint h^{N_{0}} \partial_{x}^{\alpha} \tilde{g} \partial_{\xi}^{\alpha} \psi_{1 i j}(x, D) \psi_{2 l k}\left(x, h_{k} D\right) \tilde{\chi}_{3} h^{N_{3}},
\end{aligned}
$$

with $\partial_{x}^{\alpha} \tilde{g}=\sum_{\left|\alpha_{1}\right|+\left|\alpha_{2}\right|=|\alpha|} C \partial_{x}^{\alpha_{1}}\left(\chi_{1}\left(N^{-2+} \triangle\right) u^{N_{1}}\right) \overline{\partial_{x}^{\alpha_{2}} \chi_{1}\left(N^{-2+} \triangle\right) u^{N_{2}}}$. 
First, we will estimate the case of $|\alpha|=1$, and for the term

$$
\partial_{x}\left(\chi_{1}\left(N^{-2+} \triangle\right) u^{N_{1}}\right) \overline{\chi_{1}\left(N^{-2+} \triangle\right) u^{N_{2}}} .
$$

For this case, the kernel of $\partial_{\xi} \psi_{1 i j}(x, D) \psi_{2 l k}\left(x, h_{k} D\right)$ is

$$
K(x, z)=\frac{1}{(2 \pi)^{4}} \int_{\mathbb{R}^{6}} e^{i x \xi} e^{-i z \eta} e^{i(\eta-\xi) y} \partial_{\xi} \psi_{1 i j}(x, \xi) \psi_{2 l k}\left(y, h_{k} \eta\right) d \xi d y d \eta .
$$

We will calculate the principle part, i.e. $\partial_{\xi} \psi_{10 j}\left(x, h_{j} \xi\right) \psi_{20 k}\left(y, h_{k} \eta\right)$, other terms would be no worse than it.

First for $\psi_{20 k}\left(y, h_{k} \eta\right)$,

$$
\psi_{20 k}\left(y, h_{k} \eta\right)= \begin{cases}\chi_{2}(y) \theta_{0}\left(p_{2}\left(y, N^{-1} \eta\right)\right) m\left(p_{2}\left(y, N^{-1} \eta\right)\right) & |\eta| \lesssim N \quad k=0 \\ \chi_{2}(y) \theta\left(p_{2}\left(y, h_{j} \eta\right)\right)\left|p_{2}\left(y, h_{k} \eta\right)\right|^{\frac{1-s}{2}} & |\eta| \sim h_{k}^{-1} k \geqslant 1,\end{cases}
$$

so

$$
\left|\psi_{20 k}\left(y, h_{k} \eta\right)\right| \lesssim 1
$$

Then for $\partial_{\xi} \psi_{10 j}(x, \xi)$,

$$
\partial_{\xi} \psi_{10 j}(x, \xi)=\left\{\begin{array}{cc}
\chi_{2}(x)\left(\partial_{\xi} \theta_{0}\left(p_{2}(x, \xi)\right) m\left(p_{2}(x, \xi)\right)\right. & \\
\left.+\theta_{0}\left(p_{2}(x, \xi)\right) \partial_{\xi} m\left(p_{2}(x, \xi)\right)\right) & j=0 \\
\chi_{2}(x)\left(\partial_{\xi} \theta\left(p_{2}(x, \xi)\right) m\left(p_{2}(x, \xi)\right)\right. & \\
\left.+\theta\left(p_{2}(x, \xi)\right) \partial_{\xi}\left(\left|p_{2}(x, \xi)\right|^{-\frac{1-s}{2}}\right)\right) & j \geqslant 1 .
\end{array}\right.
$$

Hence,

$$
\left|\partial_{\xi} \psi_{10 j}(x, \xi)\right| \lesssim 1
$$

By these estimates,

$$
\int|K(x, z)| d x \lesssim 1, \int|K(x, z)| d z \lesssim 1
$$

then by Schur's Lemma

$$
\left\|\partial_{\xi} \psi_{1 i j}(x, D) \psi_{2 l k}\left(x, h_{k} D\right) \tilde{\chi}_{3} h^{N_{3}}\right\|_{X_{\delta}^{0, \frac{1}{2}+}} \lesssim\left\|h^{N_{3}}\right\|_{X_{\delta}^{0, \frac{1}{2}+}} \cdot
$$

Therefore,

$$
\begin{aligned}
& \mid \int_{0}^{\delta} \int \bar{h}^{N_{0}} \partial_{x}\left(\chi_{1}\left(N^{-2+} \triangle\right) u^{N_{1}}\right) \overline{\chi_{1}\left(N^{-2+} \triangle\right) u^{N_{2}}} \\
& \qquad \partial_{\xi} \psi_{1 i j}(x, D) \psi_{2 l k}\left(x, h_{k} D\right) \tilde{\chi}_{3} h^{N_{3}} \mid \\
& \text { томе } 138-2010-\mathrm{N}^{\text {o }} 4
\end{aligned}
$$




$$
\begin{aligned}
& \lesssim \| h^{N_{0}} \partial_{x}\left(\chi_{1}\left(N^{-2+} \triangle\right) u^{N_{1}}\right) \\
& \text { - }\left\|_{L_{t, x}^{2}}\right\| \chi_{1}\left(N^{-2+} \triangle\right) u^{N_{2}} \partial_{\xi} \psi_{1 i j}(x, D) \psi_{2 l k}\left(x, h_{k} D\right) \tilde{\chi}_{3} h^{N_{3}} \|_{L_{t, x}^{2}} \\
& \lesssim N_{1}^{s_{0}+}\left\|h^{N_{0}}\right\|_{X_{\delta}^{0, \frac{1}{2}+}}\left\|\partial_{x}\left(\chi_{1}\left(N^{-2+} \triangle\right) u^{N_{1}}\right)\right\|_{X_{\delta}^{0, \frac{1}{2}+}} \\
& N_{2}^{1-}\left\|\chi_{1}\left(N^{-2+} \triangle\right) u^{N_{2}}\right\|_{X_{\delta}^{0, \frac{1}{4}+}}\left\|\partial_{\xi} \psi_{1 i j}(x, D) \psi_{2 l k}\left(x, h_{k} D\right) \tilde{\chi}_{3} h^{N_{3}}\right\|_{X_{\delta}^{0, \frac{1}{4}+}} \\
& \lesssim N^{s_{0}+} N_{1}^{0-}\left\|h^{N_{0}}\right\|_{X_{\delta}^{0, \frac{1}{2}+}}\|I u\|_{X_{\delta}^{1, \frac{1}{2}+}}^{2} \\
& \cdot \delta^{2 \times\left(\frac{1}{2}-\frac{1}{4}\right)-} N_{2}^{0-}\left\|\partial_{\xi} \psi_{1 i j}(x, D) \psi_{2 l k}\left(y, h_{k} D\right) \tilde{\chi}_{3} h^{N_{3}}\right\|_{X_{\delta}^{0, \frac{1}{2}+}} \\
& \lesssim N^{s_{0}+}\left(N_{1} N_{2}\right)^{0-} \delta^{\frac{1}{2}-}\|I u\|_{X_{\delta}^{1, \frac{1}{2}+}}^{2}\left\|h^{N_{0}}\right\|_{X_{\delta}^{0, \frac{1}{2}+}}\left\|h^{N_{3}}\right\|_{X_{\delta}^{0, \frac{1}{2}+}} \cdot
\end{aligned}
$$

Then, for $|\alpha|>1$, we could estimate similarly, since

$$
\left\|\partial_{x}^{\alpha_{1}} \chi_{1}\left(N^{-2+} \triangle\right) I u^{N_{i}}\right\|_{X_{\delta}^{1, \frac{1}{2}+}} \lesssim N^{\left|\alpha_{1}\right|-}\left\|\chi_{1}\left(N^{-2+} \triangle\right) I u^{N_{i}}\right\|_{X_{\delta}^{1, \frac{1}{2}+}},\left|\alpha_{1}\right| \geqslant 1
$$

and $h_{j}^{|\alpha|} N^{|\alpha|-}=2^{-\frac{j}{2}|\alpha|} N^{0-}$.

Conclusively,

$$
\begin{aligned}
|(8.24)| \lesssim & \delta^{\frac{1}{2}-} \sum_{j} \sum_{|k-j| \leqslant \nu} \sum_{i, l=0}^{K-1} \sum_{|\alpha|=1}^{K^{\prime}-1} 2^{\frac{k-j}{2}(1-s)-\frac{j}{2}|\alpha|} N^{-1+s_{0}+} \\
& \cdot\|I u\|_{X_{\delta}^{1, \frac{1}{2}+}}^{2} \sum_{N_{0} \sim N_{3}}\left\|h^{N_{0}}\right\|_{X_{\delta}^{0, \frac{1}{2}+}\left\|h^{N_{3}}\right\|_{X_{\delta}^{0, \frac{1}{2}+}}} \\
\lesssim & N^{-1+s_{0}+} \delta^{\frac{1}{2}-}\|I u\|_{X_{\delta}^{1, \frac{1}{2}}+}^{2} \sum_{N_{0}}\left\|h^{N_{0}}\right\|_{X_{\delta}^{0, \frac{1}{2}+}}^{2} \\
\lesssim & N^{-1+s_{0}+} \delta^{\frac{1}{2}-}\|I u\|_{X_{\delta}^{1, \frac{1}{2}+}}^{4} \lesssim N^{-1+s_{0}+} \delta^{\frac{1}{2}-}\left\|v_{0}\right\|_{H^{1}}^{4} .
\end{aligned}
$$

Therefore, combining the results of section 8.2.1 and section 8.2.2, i.e. (8.21) and (8.27), gives the result of Lemma 5.1.

Acknowledgment. - The author would like to thank her advisor Prof. D. Fang's guidance, and also her co-advisor Prof. N. Burq for his proposal to this problem and lots of discussion.

\section{BIBLIOGRAPHY}

[1] T. AKAhori - "Low regularity global well-posedness for the nonlinear Schrödinger equation on closed manifolds", Commun. Pure Appl. Anal. 9 (2010), p. 261-280. 
[2] R. Anton - "Strichartz inequalities for Lipschitz metrics on manifolds and nonlinear Schrödinger equation on domains", Bull. Soc. Math. France 136 (2008), p. 27-65.

[3] M. D. Blair, H. F. Smith \& C. D. Sogge - "On Strichartz estimates for Schrödinger operators in compact manifolds with boundary", Proc. Amer. Math. Soc. 136 (2008), p. 247-256.

[4] J. Bourgain - "Exponential sums and nonlinear Schrödinger equations", Geom. Funct. Anal. 3 (1993), p. 157-178.

[5] _ "Fourier transform restriction phenomena for certain lattice subsets and applications to nonlinear evolution equations. II. The KdVequation", Geom. Funct. Anal. 3 (1993), p. 209-262.

[6] _ ' "Refinements of Strichartz' inequality and applications to 2D-NLS with critical nonlinearity", Int. Math. Res. Not. 1998 (1998), p. 253-283.

[7] _ "A remark on normal forms and the " $I$-method" for periodic NLS", J. Anal. Math. 94 (2004), p. 125-157.

[8] _ , "On Strichartz's inequalities and the nonlinear Schrödinger equation on irrational tori", in Mathematical aspects of nonlinear dispersive equations, Ann. of Math. Stud., vol. 163, Princeton Univ. Press, 2007, p. $1-20$.

[9] N. Burq, P. GÉrard \& N. Tzvetkov - "Strichartz inequalities and the nonlinear Schrödinger equation on compact manifolds", Amer. J. Math. 126 (2004), p. 569-605.

[10] , "Bilinear eigenfunction estimates and the nonlinear Schrödinger equation on surfaces", Invent. Math. 159 (2005), p. 187-223.

[11] _ "Multilinear eigenfunction estimates and global existence for the three dimensional nonlinear Schrödinger equations", Ann. Sci. École Norm. Sup. 38 (2005), p. 255-301.

[12] T. CAzEnAVE - Semilinear Schrödinger equations, Courant Lecture Notes in Math., vol. 10, New York University Courant Institute of Mathematical Sciences, 2003.

[13] J. Colliander, M. G. Grillakis \& N. Tzirakis - "Improved interaction Morawetz inequalities for the cubic nonlinear Schrödinger equation on $\mathbb{R}^{2} "$, Int. Math. Res. Not. 2007 (2007).

[14] J. Colliander, M. Keel, G. Staffilani, H. Takaoka \& T. Tao - "Almost conservation laws and global rough solutions to a nonlinear Schrödinger equation", Math. Res. Lett. 9 (2002), p. 659-682.

[15] _ "Resonant decompositions and the $I$-method for the cubic nonlinear Schrödinger equation on $\mathbb{R}^{2 ",}$ Discrete Contin. Dyn. Syst. 21 (2008), p. 665-686. 
[16] D. De Silva, N. Pavlović, G. Staffilani \& N. Tzirakis - "Global well-posedness for a periodic nonlinear Schrödinger equation in $1 \mathrm{D}$ and 2D", Discrete Contin. Dyn. Syst. 19 (2007), p. 37-65.

[17] Y. F. FANG \& M. G. GRILLAKIS - "On the global existence of rough solutions of the cubic defocusing Schrödinger equation in $\mathbf{R}^{2+1}$ ", J. Hyperbolic Differ. Equ. 4 (2007), p. 233-257.

[18] J. GinibRE - "Le problème de Cauchy pour des équations aux dérivées partielles semi-linéaires périodiques en variables d'espace", Séminaire Bourbaki (1995), exposé $\mathrm{n}^{\circ} 796$.

[19] R. Killip, T. TAO \& M. Visan - "The cubic nonlinear Schrödinger equation in two dimensions with radial data", J. Eur. Math. Soc. (JEMS) 11 (2009), p. 1203-1258.

[20] A. MARTINEZ - An introduction to semiclassical and microlocal analysis, Universitext, Springer, 2002.

[21] S. ZHONG - "The growth in time of higher Sobolev norms of solutions to Schrödinger equations on compact Riemannian manifolds", J. Differential Equations 245 (2008), p. 359-376. 